\title{
Two conjectures by Zhi-Hong Sun
}

\author{
by
}

\author{
Constantin N. Beli (Bucureşti)
}

Let $\varepsilon$ be an algebraic integer in $\mathbb{Q}(\sqrt{d})$, where $d>1$ is square-free, and let $p>2$ be a prime with $p \nmid d$ and $p \nmid \mathrm{N}(\varepsilon)$, where $\mathrm{N}: \mathbb{Q}(\sqrt{d}) \rightarrow \mathbb{Q}$ is the norm map. It is well known that $\varepsilon^{p-1} \equiv 1(\bmod p)$ if $p \equiv 1(\bmod 4)$ and $\varepsilon^{p+1} \equiv \mathrm{N}(\varepsilon)(\bmod p)$ if $p \equiv 3(\bmod 4)$.

The next problem is to find $\varepsilon^{(p \pm 1) / 2} \bmod p$. If $\left(\frac{d}{p}\right)=\left(\frac{\mathrm{N}(\varepsilon)}{p}\right)=1$ then $\left(\frac{\varepsilon}{p}\right)$ is defined and we have $\varepsilon^{(p-1) / 2} \equiv\left(\frac{\varepsilon}{p}\right)(\bmod p)$ so the problem is equivalent to finding the Legendre symbol $\left(\frac{\varepsilon}{p}\right)$. A particular case with a long history is when $\varepsilon=\varepsilon_{d}$, the fundamental unit of $\mathbb{Q}(\sqrt{d})$ and $\mathrm{N}\left(\varepsilon_{d}\right)=-1$. The problem of finding $\left(\frac{\varepsilon_{d}}{p}\right)$ when $\mathrm{N}\left(\varepsilon_{d}\right)=-1$ for primes $p$ with $\left(\frac{-1}{p}\right)=\left(\frac{d}{p}\right)=1$ was first considered in 1942, when Aigner and Reichardt showed that if $p \equiv 1(\bmod 8)$ then $\varepsilon_{2}=1+\sqrt{2}$ is a quadratic residue modulo $p$ if and only if $p=x^{2}+32 y^{2}$ for some $x, y \in \mathbb{Z}$. Various mathematicians have obtained similar results for other fundamental units $\varepsilon_{d}$ of norm -1 . The problem was finally settled by Z. H. Sun [S1], who determined the value of $\varepsilon^{(p-(-1 / p)) / 2} \bmod p$, where $\varepsilon$ is an arbitrary integer in $\mathbb{Q}(\sqrt{d})$, in the case when $\left(\frac{-d}{p}\right)=1$. Sun's result, just as the results obtained before him, is given in terms of $x, y$ satisfying $f(x, y)=p$, where $f=A X^{2}+2 B X Y+C Y^{2}$ is a quadratic form with $\operatorname{det} f=B^{2}-A C=-k^{2} d$ and $k$ is a (bounded) positive integer. The fact that $p$ can be represented by one of these quadratic forms is ensured by the fact that $\left(\frac{-d}{p}\right)=1$.

The next level of difficulty is to calculate $\varepsilon^{(p-(-1 / p)) / 4} \bmod p$, again when $\left(\frac{-d}{p}\right)=1$. In this paper we restrict ourselves to the case when $p \equiv 3$ $(\bmod 4)$, i.e. $\left(\frac{-1}{p}\right)=\left(\frac{d}{p}\right)=-1$, and we determine $\varepsilon^{(p+1) / 4} \bmod p$. We solve two conjectures by Z. H. Sun regarding the value of $\varepsilon_{5}^{(p+1) / 4} \bmod p$, where $\varepsilon_{5}=(1+\sqrt{5}) / 2$, for $p \equiv 3,7(\bmod 20)$, and the value of $\varepsilon_{3}^{(p+1) / 8} \bmod p$, where $\varepsilon_{3}=2+\sqrt{3}$, for $p \equiv 7(\bmod 24)$. Apparently the second conjec- 
ture is one level of difficulty up because of the denominator 8. However, if we note that $2+\sqrt{3}=(1+\sqrt{3})^{2} / 2$ then the problem reduces to finding $(1+\sqrt{3})^{(p+1) / 4} \bmod p$.

The conjecture regarding $(2+\sqrt{3})^{(p+1) / 8}$ is related to a problem involving the sequence $S_{k}$ given by $S_{1}=4, S_{k+1}=S_{k}^{2}-2$, from the Lucas-Lehmer test, which is mentioned in [G, A3]. If $M_{p}=2^{p}-1$ is a Mersenne prime then $p \mid S_{p-1}=S_{p-2}^{2}-2$ so $S_{p-2}^{2} \equiv 2 \equiv 2^{p+1}\left(\bmod 2^{p}-1\right)$, so $S_{p-2} \equiv$ $\pm 2^{(p+1) / 2}\left(\bmod M_{p}\right)$. The problem is to determine the \pm sign. We have $S_{k}=(2+\sqrt{3})^{2^{k-1}}+(2-\sqrt{3})^{2^{k-1}}=2 V_{2^{k-1}}$, where $V_{n}$ is given by the formula $V_{n}+U_{n} \sqrt{3}=(2+\sqrt{3})^{n}$. Therefore we want to know the \pm sign for which $2 V_{\left(M_{p}+1\right) / 8}=2 V_{2^{p-3}}=S_{p-2} \equiv \pm 2^{(p+1) / 2}\left(\bmod M_{p}\right)$. Since $M_{p} \equiv 7(\bmod 8)$ we have $\left(\frac{-1}{M_{p}}\right)=-1$ and $\left(\frac{2}{M_{p}}\right)=1$. Thus the $\pm \operatorname{sign}$ equals $\left(\frac{S_{p-2}}{M_{p}}\right)=$ $\left(\frac{V_{\left(M_{p}+1\right) / 8}}{M_{p}}\right)$. Sun's conjecture gives the quadratic residues mod $p$ for both $V_{(p+1) / 8}$ and $U_{(p+1) / 8}$ for any $p \equiv 7(\bmod 24)$ and it allows us to determine $(2+\sqrt{3})^{(p+1) / 8} \bmod p$. As a consequence, our $\pm \operatorname{sign}$ is $(-1)^{\left(x^{2}-4\right) / 32}$, where $M_{p}=x^{2}+3 y^{2}$ with $x, y \in \mathbb{Z}$.

The methods we use are from class field theory. Given a number field $F$ and a (possibly archimedian) prime $\mathfrak{p}$ of $F$, for any $x \in F$ we denote by $x_{\mathfrak{p}}$ its image in $F_{\mathfrak{p}}$. When there is no danger of confusion we simply write $x$ instead of $x_{\mathfrak{p}}$. If $E / F$ is a finite abelian extension and $\mathfrak{P}$ is a prime of $E$ lying over $\mathfrak{p}$ then we denote by $\left(\frac{\cdot E / F}{\mathfrak{p}}\right): F_{\mathfrak{p}}^{\times} \rightarrow \operatorname{Gal}(E / F)$ the Artin map and by $\left(\cdot, E_{\mathfrak{P}} / F_{\mathfrak{p}}\right): F_{\mathfrak{p}}^{\times} \rightarrow \operatorname{Gal}\left(E_{\mathfrak{P}} / F_{\mathfrak{p}}\right)$ the local Artin map. If we identify $\operatorname{Gal}\left(E_{\mathfrak{P}} / F_{\mathfrak{p}}\right)$ with its image in $\operatorname{Gal}(E / F)$ then $\left(\frac{a, E / F}{\mathfrak{p}}\right)=\left(a, E_{\mathfrak{P}} / F_{\mathfrak{p}}\right)$ for any $a \in F_{\mathfrak{p}}^{\times}$.

1. $F_{(p+1) / 4}$ and $L_{(p+1) / 4} \bmod p$ for $p \equiv 3,7(\bmod 20)$. Let $F_{n}, L_{n}$ be the Fibonacci and Lucas sequences given by $F_{0}=0, F_{1}=1$ and $F_{n+1}=$ $F_{n-1}+F_{n}$ and by $L_{0}=2, L_{1}=1$ and $L_{n+1}=L_{n-1}+L_{n}$. We have

$$
\frac{L_{n}+F_{n} \sqrt{5}}{2}=\left(\frac{1+\sqrt{5}}{2}\right)^{n} \text {. }
$$

In [S1, Conjecture 5.2] Z. H. Sun proposed the following conjecture:

Conjecture 1.1 (Z. H. Sun, 2003). If $p \equiv 3,7(\bmod 20)$ is a prime with $2 p=x^{2}+5 y^{2}$ for some integers $x$, $y$ then $F_{(p+1) / 4} \equiv \begin{cases}2(-1)^{[(p-5) / 10]} 10^{(p-3) / 4}(\bmod p) & \text { if } y \equiv \pm(p-1) / 2(\bmod 8), \\ -2(-1)^{[(p-5) / 10]} 10^{(p-3) / 4}(\bmod p) & \text { if } y \neq \equiv \pm(p-1) / 2(\bmod 8) .\end{cases}$

By [SS, Corollary 2(iii)] we have

$$
F_{(p+1) / 4} L_{(p+1) / 4}=F_{(p+1) / 2} \equiv 2(-1)^{[(p-5) / 10]} 5^{(p-3) / 4}(\bmod p) .
$$


Therefore the conjecture above is equivalent to:

$$
L_{(p+1) / 4} \equiv \begin{cases}(-2)^{(p+1) / 4}(\bmod p) & \text { if } y \equiv \pm(p-1) / 2(\bmod 8), \\ -(-2)^{(p+1) / 4}(\bmod p) & \text { if } y \neq \equiv(p-1) / 2(\bmod 8) .\end{cases}
$$

We have $p \equiv 3(\bmod 4)$ and $p \equiv 2,3(\bmod 5)$ so $\left(\frac{-1}{p}\right)=\left(\frac{5}{p}\right)=-1$.

1.2. Since $\left(L_{n}+F_{n} \sqrt{5}\right) / 2=\varepsilon^{n}$, where $\varepsilon:=(1+\sqrt{5}) / 2$, we have to evaluate $\varepsilon^{(p+1) / 4} \bmod p$. We have $\left(\frac{5}{p}\right)=-1$ so $p$ is inert in $\mathbb{Q}(\sqrt{5})$. Since $p \nmid \varepsilon$ the conjugate of $\varepsilon, \bar{\varepsilon}:=(1-\sqrt{5}) / 2$, is given by the Frobenius automorphism $\bar{\varepsilon}=\Phi_{p}(\varepsilon) \equiv \varepsilon^{p}(\bmod p)$. Thus $\varepsilon^{p+1} \equiv \varepsilon \bar{\varepsilon}=-1(\bmod p)$.

Note that $(p-1) / 2$ is odd so

$$
\left(p+1, \frac{p^{2}-1}{8}\right)=\frac{p+1}{4}\left(4, \frac{p-1}{2}\right)=\frac{p+1}{4} .
$$

Thus if we also know $\varepsilon^{\left(p^{2}-1\right) / 8} \bmod p$ then we know $\varepsilon^{(p+1) / 4} \bmod p$. More precisely, if $p \equiv 3(\bmod 8)$ then

so

$$
\frac{p+1}{4}=\frac{p^{2}-1}{8}-(p+1) \cdot \frac{p-3}{8}
$$

$$
\varepsilon^{(p+1) / 4}=\varepsilon^{\left(p^{2}-1\right) / 8}\left(\varepsilon^{p+1}\right)^{-(p-3) / 8} \equiv(-1)^{(p-3) / 8} \varepsilon^{\left(p^{2}-1\right) / 8}(\bmod p),
$$

while if $p \equiv 7(\bmod 8)$ then

so

$$
\frac{p+1}{4}=-\frac{p^{2}-1}{8}+(p+1) \cdot \frac{p+1}{8}
$$

$$
\varepsilon^{(p+1) / 4}=\varepsilon^{-\left(p^{2}-1\right) / 8}\left(\varepsilon^{p+1}\right)^{(p+1) / 8} \equiv(-1)^{(p+1) / 8} \varepsilon^{-\left(p^{2}-1\right) / 8}(\bmod p) .
$$

We will obtain $\varepsilon^{\left(p^{2}-1\right) / 8} \bmod p$ in terms of some Hilbert symbol of order 8 . To do this we will construct a cyclic extension of order 8 of $F:=$ $\mathbb{Q}(\sqrt{5})$.

Since $\left(\frac{-1}{p}\right)=-1$ we have either $\left(\frac{2}{p}\right)=1($ if $p \equiv 7(\bmod 8))$ or $\left(\frac{-2}{p}\right)=1$ (if $p \equiv 3(\bmod 8))$. This implies that we can write $2 p=u^{2}-2 v^{2}$ if $p \equiv 7$ $(\bmod 8)$ or $2 p=u^{2}+2 v^{2}$ if $p \equiv 3(\bmod 8)$.

Let $F=\mathbb{Q}(\sqrt{5})$ and $E=F(\zeta)=F(i, \sqrt{2})$, where $\zeta:=\zeta_{8}=(1+i) / \sqrt{2}$. Note that the morphisms $\zeta \mapsto \zeta^{k}$ with $k \in \mathbb{Z}_{8}^{\times}$behave as follows: $\zeta \mapsto \zeta$ is the identity; $\zeta \mapsto \zeta^{3}$ is given by $i \mapsto-i$ and $\sqrt{2} \mapsto-\sqrt{2} ; \zeta \mapsto \zeta^{5}$ is given by $i \mapsto i$ and $\sqrt{2} \mapsto-\sqrt{2} ; \zeta \mapsto \zeta^{7}$ is given by $i \mapsto-i$ and $\sqrt{2} \mapsto \sqrt{2}$.

Define $A_{1} \in E$ by

$$
A_{1}= \begin{cases}2 p(x+y \sqrt{5} i)^{2}(u+v \sqrt{2} i)^{4} & \text { if } p \equiv 3(\bmod 8) \\ 2 p(x+y \sqrt{5} i)^{6}(u+v \sqrt{2})^{4} & \text { if } p \equiv 7(\bmod 8)\end{cases}
$$


Note that $\sqrt{2 p} \notin E=\mathbb{Q}(i, \sqrt{5}, \sqrt{2})$ so $A_{1}$ is not a square in $E$. For $k \in \mathbb{Z}_{8}^{\times}$ we denote by $A_{k}$ the image of $A_{1}$ under the automorphism $\zeta \mapsto \zeta^{k}$ from $\operatorname{Gal}(E / F)$.

Let $L=E\left(\sqrt[8]{A_{1}}\right)$. Since $\mu_{8} \subset E$ and $A_{1}$ is not a square in $E$ we have $\operatorname{Gal}(L / E)=\langle\sigma\rangle \cong \mathbb{Z}_{8}$, where $\sigma \in \operatorname{Gal}(L / E)$ is given by $\sqrt[8]{A_{1}} \mapsto \zeta \sqrt[8]{A_{1}}$.

Lemma 1.3. The extension $L / F$ is Galois and $\operatorname{Gal}(L / F) \cong \mathbb{Z}_{8}^{\times} \times \mathbb{Z}_{8}$.

Proof. First we prove that $L / F$ is normal. Define $\alpha_{1}=\sqrt[8]{A_{1}}$. Let $\bar{F}$ be some algebraic closure of $F$ containing $L$ and let $\alpha \in \bar{F}$ be some conjugate of $\alpha_{1}$ over $F$. Then $\alpha^{8}$ is a conjugate of $\alpha_{1}^{8}=A_{1}$ over $F$ so it is of the form $A_{k}$ with $k \in \mathbb{Z}_{8}^{\times}$. We show that for any $k \in \mathbb{Z}_{8}^{\times}, k \neq 1$, we have $A_{k}=\alpha_{k}^{8}$, where

$$
\begin{gathered}
\alpha_{3}= \begin{cases}\alpha_{1}^{3}(x+y \sqrt{5} i)^{-1}(u+v \sqrt{2} i)^{-1} & \text { if } p \equiv 3(\bmod 8), \\
\alpha_{1}^{3} \cdot 2 p(x+y \sqrt{5} i)^{-3}(u+v \sqrt{2})^{-2} & \text { if } p \equiv 7(\bmod 8),\end{cases} \\
\alpha_{5}= \begin{cases}\alpha_{1}^{5}(x+y \sqrt{5} i)^{-1}(u+v \sqrt{2} i)^{-3} & \text { if } p \equiv 3(\bmod 8), \\
\alpha_{1}^{5}(x+y \sqrt{5} i)^{-3}(u+v \sqrt{2})^{-3} & \text { if } p \equiv 7(\bmod 8),\end{cases} \\
\alpha_{7}= \begin{cases}2 p \alpha_{1}^{-1}=\alpha_{1}^{7}(x+y \sqrt{5} i)^{-2}(u+v \sqrt{2} i)^{-4} & \text { if } p \equiv 3(\bmod 8), \\
2 p(u+v \sqrt{2}) \alpha_{1}^{-1}=\alpha_{1}^{7}(x+y \sqrt{5} i)^{-6}(u+v \sqrt{2})^{-3} & \text { if } p \equiv 7(\bmod 8) .\end{cases}
\end{gathered}
$$

The proof is straightforward and it uses the relations $\alpha_{1}^{8}=A_{1}, 2 p=$ $(x+y \sqrt{5} i)(x-y \sqrt{5} i)$ and $2 p=(u+v \sqrt{2} i)(u-v \sqrt{2} i)$ or $(u+v \sqrt{2})(u-v \sqrt{2})$, corresponding to $p \equiv 3(\bmod 8)$ or $p \equiv 7(\bmod 8)$, respectively, and also the way the morphisms $\zeta \mapsto \zeta^{k}$ from $\operatorname{Gal}(E / F)$ act on $\sqrt{2}$ and $i$. For illustration we give the argument for $\alpha_{5}$ when $p \equiv 3(\bmod 8)$. Since the morphism $\zeta \mapsto \zeta^{5}$ is given by $i \mapsto i$ and $\sqrt{2} \mapsto-\sqrt{2}$ we have

$$
\begin{aligned}
A_{5} & =2 p(x+y \sqrt{5} i)^{2}(u-v \sqrt{2} i)^{4}=(2 p)^{5}(x+y \sqrt{5} i)^{2}(u+v \sqrt{2} i)^{-4} \\
& =A_{1}^{5}(x+y \sqrt{5} i)^{-8}(u+v \sqrt{2} i)^{-24}=\alpha_{3}^{8},
\end{aligned}
$$

where $\alpha_{5}=\alpha_{1}^{5}(x+y \sqrt{5} i)^{-1}(u+v \sqrt{2} i)^{-3}$.

In all cases we have $\alpha^{8}=A_{k}=\alpha_{k}^{8}$ for some $\alpha_{k} \in L$, with $k \in \mathbb{Z}_{8}^{\times}$. Therefore $\alpha=\zeta^{l} \alpha_{k}$ for some $l$ so $\alpha \in L$. So the 32 conjugates of $\alpha_{1}$ over $F$ are $\zeta^{l} \alpha_{k}$ with $k \in \mathbb{Z}_{8}^{\times}$and $l \in \mathbb{Z}_{8}$.

Let $\phi \in \operatorname{Gal}(L / F)$. Then $\phi_{\mid E}$ is of the form $\zeta \mapsto \zeta^{k}$ with $k \in \mathbb{Z}_{8}^{\times}$. It follows that $\phi\left(\alpha_{1}\right)^{8}=\phi\left(A_{1}\right)=A_{k}=\alpha_{k}^{8}$ so $\phi\left(\alpha_{1}\right)=\zeta^{l} \alpha_{k}$ for some $l$. Therefore the 32 elements of $\operatorname{Gal}(L / F)$ are given by $\zeta \mapsto \zeta^{k}, \alpha_{1} \mapsto \zeta^{l} \alpha_{k}$ with $k \in \mathbb{Z}_{8}^{\times}$, $l \in \mathbb{Z}_{8}$. For $k \in \mathbb{Z}_{8}^{\times}$we denote by $\tau_{k}$ the morphism $\zeta \mapsto \zeta^{k}, \alpha_{1} \mapsto \alpha_{k}$.

Let $H=\left\{\tau_{k} \mid k \in \mathbb{Z}_{8}^{\times}\right\}$. We now prove that $H$ is a subgroup of $\operatorname{Gal}(L / F)$, the mapping $k \mapsto \tau_{k}$ defines an isomorphism between $\mathbb{Z}_{8}^{\times}$and $H$, and $\operatorname{Gal}(L / F)$ is the internal direct product of its subgroups $H$ and $\operatorname{Gal}(L / E)=$ $\langle\sigma\rangle$. To do this we have to prove that $H \cap \operatorname{Gal}(L / E)=\{1\}, \tau_{k} \sigma=\sigma \tau_{k}$ for 
$k \in \mathbb{Z}_{8}^{\times}$, and $\tau_{k} \tau_{l}=\tau_{k l}$ for $k, l \in \mathbb{Z}_{8}^{\times}$. (In fact, for the second assertion we only need to prove that $\tau_{3}^{2}=\tau_{5}^{2}=\tau_{7}^{2}=1$ and $\tau_{3} \tau_{5}=\tau_{7}$.)

If $\phi \in H \cap \operatorname{Gal}(L / E)$ then $\phi=\tau_{k}=\sigma^{l}$ for some $k \in \mathbb{Z}_{8}^{\times}$and $l \in \mathbb{Z}_{8}$. It follows that $\alpha_{k}=\tau_{k}\left(\alpha_{1}\right)=\sigma^{l}\left(\alpha_{1}\right)=\zeta^{l} \alpha_{1}$ so $A_{k}=\alpha_{k}^{8}=\left(\zeta^{l} \alpha_{1}\right)^{8}=A_{1}$. Hence $k=1$ and we have $\phi=\tau_{1}=1$.

For the second assertion we note that in all cases we have $\alpha_{k}=\alpha_{1}^{k} a$ for some $a \in E$. Now $\tau_{k} \sigma(\zeta)=\tau_{k}(\zeta)=\zeta^{k}$ and $\sigma \tau_{k}(\zeta)=\sigma\left(\zeta^{k}\right)=\zeta^{k}$. Also $\tau_{k} \sigma\left(\alpha_{1}\right)=\tau_{k}\left(\zeta \alpha_{1}\right)=\zeta^{k} \alpha_{k}$ and $\sigma \tau_{k}\left(\alpha_{1}\right)=\sigma\left(\alpha_{k}\right)=\sigma\left(\alpha_{1}^{k} a\right)=\left(\zeta \alpha_{1}\right)^{k} a=$ $\zeta^{k} \alpha_{k}$. So $\tau_{k} \sigma=\sigma \tau_{k}$.

The proof of the third assertion is more laborious as it involves many cases. We first prove that $\tau_{3} \tau_{5}=\tau_{7}$ when $p \equiv 3(\bmod 8)$. Since $\tau_{k}(\zeta)=\zeta^{k}$ we have $\tau_{3} \tau_{5}(\zeta)=\zeta^{15}=\zeta^{7}=\tau_{7}(\zeta)$. Also

$$
\begin{aligned}
\tau_{3} \tau_{5}\left(\alpha_{1}\right) & =\tau_{3}\left(\alpha_{5}\right)=\tau_{3}\left(\alpha_{1}^{5}(x+y \sqrt{5} i)^{-1}(u+v \sqrt{2} i)^{-3}\right) \\
& =\alpha_{3}^{5}(x-y \sqrt{5} i)^{-1}(u+v \sqrt{2} i)^{-3} \\
& =\left(\alpha_{1}^{3}(x+y \sqrt{5} i)^{-1}(u+v \sqrt{2} i)^{-1}\right)^{5}(2 p)^{-1}(x+y \sqrt{5} i)(u+v \sqrt{2} i)^{-3} \\
& =\alpha_{1}^{15}(2 p)^{-1}(x+y \sqrt{5} i)^{-4}(u+v \sqrt{2} i)^{-8} \\
& =\alpha_{1}^{7}(x+y \sqrt{5} i)^{-2}(u+v \sqrt{2} i)^{-4}=\alpha_{7}=\tau_{7}\left(\alpha_{1}\right) .
\end{aligned}
$$

So $\tau_{3} \tau_{5}=\tau_{7}$. (Recall that $\tau_{3 \mid E}$ is given by $\zeta \mapsto \zeta^{3}$, i.e. by $i \mapsto-i, \sqrt{2} \mapsto-\sqrt{2}$. Also $\alpha_{1}^{8}=A_{1}=2 p(x+y \sqrt{5} i)^{2}(u+v \sqrt{2} i)^{4}$.)

The proof of $\tau_{k} \tau_{l}=\tau_{k l}$ in all the other cases is quite straightforward if we follow the pattern above. The reason why it always works is the following: We have $\tau_{k} \tau_{l}(\zeta)=\tau_{k}\left(\zeta^{l}\right)=\zeta^{k l}$. As seen above, this implies that $\tau_{k} \tau_{l}\left(\alpha_{1}\right)$ is of the form $\zeta^{j} \alpha_{k l}$ for some $j$. But in calculating $\tau_{k} \tau_{l}\left(\alpha_{1}\right)$, as well as $\tau_{k l}\left(\alpha_{1}\right)=\alpha_{k l}$, $\zeta$ is not involved. (We only have the factors $x \pm y \sqrt{5} i, u \pm v \sqrt{2} i$ (if $p \equiv 3$ $(\bmod 8)), u \pm v \sqrt{2}($ if $p \equiv 7(\bmod 8)), 2 p$ and $\alpha_{1}$.) Therefore we must have $j=0$. Hence $\tau_{k} \tau_{l}$ is given by $\zeta \mapsto \zeta^{k l}, \alpha_{1} \mapsto \alpha_{k l}$ so it is equal to $\tau_{k l}$.

Consequently, $\operatorname{Gal}(L / F)=H \times \operatorname{Gal}(L / E) \cong \mathbb{Z}_{8}^{\times} \times \mathbb{Z}_{8}$.

Let $K=L^{H}$. Then $\operatorname{Gal}(K / F) \cong \operatorname{Gal}(L / F) / H=\langle\sigma H\rangle \cong \mathbb{Z}_{8}$, the isomorphism being given by $\phi_{\mid K} \mapsto \phi H$. We denote by $\chi_{0}: \operatorname{Gal}(K / F) \rightarrow \mu_{8}$ the isomorphism given by $\sigma_{\mid K}^{k} \mapsto \zeta^{k}$. It induces a character $\chi: \operatorname{Gal}(L / F) \rightarrow \mu_{8}$ given by the composition with $\operatorname{Gal}(L / F) \rightarrow \operatorname{Gal}(K / F)$. For any $\sigma^{k} \tau_{l} \in$ $\operatorname{Gal}(L / F)$ we have $\chi\left(\sigma^{k} \tau_{l}\right)=\zeta^{k}$. We obtain

$$
\prod_{\mathfrak{q}}\left(\frac{\varepsilon, L / F}{\mathfrak{q}}\right)=1 \quad \text { so } \quad \chi\left(\prod_{\mathfrak{q}}\left(\frac{\varepsilon, L / F}{\mathfrak{q}}\right)\right)=1 .
$$

Note that $\sigma^{k}\left(\alpha_{1}\right)=\zeta^{k} \alpha_{1}$ so for any $\phi \in\langle\sigma\rangle=\operatorname{Gal}(L / E)$ we have $\chi(\phi)=\phi\left(\alpha_{1}\right) / \alpha_{1}$. 
Since $\left(\frac{5}{p}\right)=-1$ the extension $F / \mathbb{Q}$ is inert at $p$. Let $\mathfrak{p}$ be the prime of $F$ lying over $p$, i.e. $\mathfrak{p}=p \mathcal{O}_{F}$. Now $-1,2$ are units in $\mathbb{Q}_{p}$ so they are in the square class modulo $p$ of either 1 or 5 . In both cases they are squares in $F_{\mathfrak{p}}$. Therefore the prime $\mathfrak{p}$ splits completely in $E=F(i, \sqrt{2})$. Now $(x+y \sqrt{5} i)(x-y \sqrt{5} i)=$ $2 p$ and either $(u+v \sqrt{2} i)(u-v \sqrt{2} i)=2 p$ or $(u+v \sqrt{2})(u-v \sqrt{2})=2 p$ (according as $p \equiv 3$ or $7(\bmod 8))$. So for every prime $\mathfrak{P}$ of $E$ lying over $\mathfrak{p}$ exactly one of $x \pm y \sqrt{5} i$ and exactly one of $u \pm v \sqrt{2} i$ or $u \pm v \sqrt{2}$ belongs to $\mathfrak{P}$. Of the four primes of $E$ that lie over $\mathfrak{p}$ we choose the one for which $x-y \sqrt{5} i \in \mathfrak{P}$, and $u-v \sqrt{2} i \in \mathfrak{P}$ or $u-v \sqrt{2} \in \mathfrak{P}$, corresponding to $p \equiv 3$ or $7(\bmod 8)$.

Denote by $\infty_{ \pm}$the two archimedian primes of $F$ corresponding to the embeddings $F \hookrightarrow \mathbb{R}$ given by $\sqrt{5} \mapsto \pm \sqrt{5}$.

LEMMA 1.4.

(i) $\chi\left(\left(\frac{\varepsilon, L / F}{\mathfrak{p}}\right)\right) \equiv \varepsilon^{-\left(p^{2}-1\right) / 8}(\bmod \mathfrak{P})$.

(ii) $\chi\left(\left(\frac{\varepsilon, L / F}{\infty_{-}}\right)\right)= \begin{cases}1 & \text { if } p \equiv 3(\bmod 8), \\ \operatorname{sgn}(u) & \text { if } p \equiv 7(\bmod 8) .\end{cases}$

(iii) $\chi\left(\left(\frac{\varepsilon, L / F}{\mathfrak{q}}\right)\right)=1$ if $\mathfrak{q} \neq \mathfrak{p}, \infty_{-}$and $\mathfrak{q} \nmid 2$.

Proof. (i) Since $\mathfrak{p}$ splits in $E$ we have $\left[E_{\mathfrak{P}}: F_{\mathfrak{p}}\right]=1$ so $N_{E_{\mathfrak{P}} / F_{\mathfrak{p}}} \varepsilon=\varepsilon$ so $\left(\frac{\varepsilon, L / F}{\mathfrak{p}}\right)=\left(\frac{\varepsilon, L / E}{\mathfrak{P}}\right) \in \operatorname{Gal}(L / E)$. It follows that

$$
\begin{aligned}
\chi\left(\left(\frac{\varepsilon, L / F}{\mathfrak{p}}\right)\right) & =\chi\left(\left(\frac{\varepsilon, L / E}{\mathfrak{P}}\right)\right)=\left(\frac{\varepsilon, L / E}{\mathfrak{P}}\right)\left(\alpha_{1}\right) / \alpha_{1} \\
& =\left(\frac{\varepsilon, A_{1}}{\mathfrak{P}}\right)_{8}=\left(\frac{A_{1}, \varepsilon}{\mathfrak{P}}\right)_{8}^{-1} .
\end{aligned}
$$

(Recall that $L=E\left(\alpha_{1}\right)$ and we have $\alpha_{1}^{8}=A_{1} \in E$ and $\mu_{8} \subset E$.)

Note that $\varepsilon$ is a unit in $E_{\mathfrak{P}}$ so $\left(\frac{A_{1}, \varepsilon}{\mathfrak{P}}\right)_{8} \equiv \varepsilon^{\left(\mathrm{NP}^{\text {ord }} \mathfrak{P} A_{1}-1\right) / 8}(\bmod \mathfrak{P})$. But $E_{\mathfrak{P}} \cong F_{\mathfrak{p}}$ is an unramified extension of degree 2 of $\mathbb{Q}_{p}$ so $\operatorname{ord}_{\mathfrak{P}} p=1$ and $\mathrm{NP}=p^{2}$. Since also $x+y \sqrt{5} i \notin \mathfrak{P}$ and either $u+v \sqrt{2} i \notin \mathfrak{P}$ or $u+v \sqrt{2} \notin \mathfrak{P}$, depending on whether $p \equiv 3$ or $7(\bmod 8)$, we get $\operatorname{ord}_{\mathfrak{P}} A_{1}=1$. Therefore

$$
\chi\left(\left(\frac{\varepsilon, L / F}{\mathfrak{p}}\right)\right)=\left(\frac{A_{1}, \varepsilon}{\mathfrak{P}}\right)_{8}^{-1} \equiv \varepsilon^{-\left(p^{2}-1\right) / 8}(\bmod \mathfrak{P}) .
$$

(ii) Let $\mathfrak{q}=\infty_{-}$and let $\mathfrak{Q}$ be the infinite prime of $E=F(\zeta)$ lying over $\mathfrak{q}$ corresponding to an embedding of $E$ in $\mathbb{C}$ given by $\zeta \mapsto \zeta$ (i.e. the embedding of $E=\mathbb{Q}(\sqrt{5}, i, \sqrt{2})$ in $\mathbb{C}$ given by $\sqrt{5} \mapsto-\sqrt{5}, i \mapsto i, \sqrt{2} \mapsto \sqrt{2})$. Finally, we extend $\mathfrak{Q}$ to a prime $\mathcal{Q}$ of $L$. 
We have $F_{\mathfrak{q}} \cong \mathbb{R}$ and $E_{\mathfrak{Q}} \cong L_{\mathcal{Q}} \cong \mathbb{C}$. Moreover, $\varepsilon_{\mathfrak{q}}=(1-\sqrt{5}) / 2<0$ so if $\phi=\left(\frac{\varepsilon, L / F}{\mathfrak{q}}\right)$ then $\phi$ corresponds to $c$, the conjugacy automorphism from $\operatorname{Gal}\left(L_{\mathcal{Q}} / F_{\mathfrak{q}}\right) \cong \operatorname{Gal}(\mathbb{C} / \mathbb{R})$. So we want to know what automorphism $\phi \in \operatorname{Gal}(L / F)$ corresponds to $c \in \operatorname{Gal}\left(L_{\mathcal{Q}} / F_{\mathfrak{q}}\right)$. First note that $\phi(\zeta)=c(\zeta)=$ $\bar{\zeta}=\zeta^{7}=\tau_{7}(\zeta)$. Thus $\phi_{\mid E}=\tau_{7 \mid E}$. It follows that $\phi \in \tau_{7} \operatorname{Gal}(L / E)=\tau_{7}\langle\sigma\rangle$. So $\phi=\sigma^{k} \tau_{7}$ for some $k \in \mathbb{Z}_{8}$. We have $\chi(\phi)=\zeta^{k}$.

If $p \equiv 3(\bmod 8)$ then $\phi\left(\alpha_{1}\right)=\sigma^{k} \tau_{7}\left(\alpha_{1}\right)=\sigma^{k}\left(2 p \alpha_{1}^{-1}\right)=2 p \zeta^{-k} \alpha_{1}^{-1}$. On the other hand, $\phi\left(\alpha_{1}\right)=c\left(\alpha_{1}\right)=\bar{\alpha}_{1}$. Hence $\alpha_{1} \bar{\alpha}_{1}=2 p \zeta^{-k}$. Since $\alpha_{1} \bar{\alpha}_{1} \in \mathbb{R}_{+}$ and $\zeta^{-k} \in \mu_{8}$ we must have $\zeta^{-k}=1$ so $\chi\left(\left(\frac{\varepsilon, L / F}{\mathfrak{q}}\right)\right)=\zeta^{k}=1$, as claimed. The proof in the case when $p \equiv 7(\bmod 8)$ is similar but this time $\alpha_{7}=2 p(u+$ $v \sqrt{2}) \alpha_{1}$ so we get $\alpha_{1} \bar{\alpha}_{1}=2 p(u+v \sqrt{2}) \zeta^{-k}$. So $\alpha_{1} \bar{\alpha}_{1} \in \mathbb{R}_{+}$and $\zeta^{-k} \in \mu_{8}$ will imply this time $\zeta^{-k}=\operatorname{sgn}(u+v \sqrt{2})$. Thus $\chi\left(\left(\frac{\varepsilon, L / F}{\mathfrak{q}}\right)\right)=\zeta^{k}=\operatorname{sgn}(u+v \sqrt{2})$. But $u^{2}-2 v^{2}=2 p>0$ so $|u|>|v \sqrt{2}|$. Hence $\operatorname{sgn}(u+v \sqrt{2})=\operatorname{sgn}(u)$ and we get the desired result.

(iii) If $\mathfrak{q}=\infty_{+}$then $\varepsilon_{\mathfrak{q}}=(1+\sqrt{5}) / 2>0$ so $\left(\frac{\varepsilon, L / F}{\mathfrak{q}}\right)=1_{L}$. If $\mathfrak{q}$ is non-archimedian lying over $q \neq 2, p$ then $\mathfrak{q}$ does not ramify in $E=F(\zeta)$. If $\mathfrak{Q}$ is a prime of $E$ lying over $\mathfrak{q}$ then $\mathfrak{Q}$ does not ramify in $L=E\left(\sqrt[8]{A_{1}}\right)$ because $A_{1}$ is a unit in $E_{\mathfrak{Q}}$. This happens because $A_{1}$ divides a power of $2 p$ and $(2 p, q)=1$. (If $p \equiv 3(\bmod 8)$ we have $x+y \sqrt{5} i \mid 2 p$ and $u+v \sqrt{2} i \mid 2 p$ so $A_{1}=2 p(x+y \sqrt{5} i)^{2}(u+v \sqrt{2} i)^{4}$ divides $(2 p)^{7}$. Similarly, if $p \equiv 7(\bmod 8)$ then $A_{1}=2 p(x+y \sqrt{5} i)^{6}(u+v \sqrt{2})^{4}$ divides $(2 p)^{11}$.) Hence $\mathfrak{q}$ does not ramify in $L$. Since $\varepsilon$ is a unit in $F$ we have again $\left(\frac{\varepsilon, L / F}{\mathfrak{q}}\right)=1_{L}$. Therefore $\chi\left(\left(\frac{\varepsilon, L / F}{\mathfrak{q}}\right)\right)=1$.

1.5. By Lemma 1.4 the relation $\prod_{\mathfrak{q}} \chi\left(\left(\frac{\varepsilon, L / F}{\mathfrak{q}}\right)\right)=1$ implies

$$
\varepsilon^{\left(p^{2}-1\right) / 8} \equiv \begin{cases}\chi\left(\left(\frac{\varepsilon, L / F}{\mathfrak{q}}\right)\right)(\bmod \mathfrak{P}) & \text { if } p \equiv 3(\bmod 8), \\ \operatorname{sgn}(u) \chi\left(\left(\frac{\varepsilon, L / F}{\mathfrak{q}}\right)\right)(\bmod \mathfrak{P}) & \text { if } p \equiv 7(\bmod 8)\end{cases}
$$

where $\mathfrak{q}$ is the only prime of $F$ lying over 2 . (The prime 2 is inert in $F=$ $\mathbb{Q}(\sqrt{5})$.

Unfortunately, calculating the local Artin map $\left(\frac{\varepsilon, L / F}{\mathfrak{q}}\right)$ is very difficult since $\mathfrak{q}$ ramifies in $L$. In order to circumvent this impediment we show that if $p^{\prime} \equiv 3,7(\bmod 20)$ is another prime and $x^{\prime}, y^{\prime}, u^{\prime}, v^{\prime}, L^{\prime}, \chi^{\prime}$ are the $x, y, u, v, L, \chi$ corresponding to $p^{\prime}$, and $p, x, y, u, v$ are close enough to $p^{\prime}, x^{\prime}, y^{\prime}, u^{\prime}, v^{\prime}$ in the 2-adic topology, then $\chi\left(\left(\frac{\varepsilon, L / F}{\mathfrak{q}}\right)\right)=\chi\left(\left(\frac{\varepsilon, L^{\prime} / F}{\mathfrak{q}}\right)\right)$. Next we show that if $p \equiv p^{\prime}(\bmod 16)$ and $y \equiv \pm y^{\prime}(\bmod 8)$, then our conjecture is true for $p$ iff it is true for $p^{\prime}$. Hence we reduce the proof to a finite number of $p$ 's. 
LEMMA 1.6. Let $k$ be a finite extension of $\mathbb{Q}_{2}, \mathcal{O}$ its ring of integers, $\mathfrak{m}$ the maximal ideal of $\mathcal{O}$, and $\mathcal{O}^{\times}=\mathcal{O} \backslash \mathfrak{m}$ the group of units. Let $j \geq 1$.

(i) If $\alpha \in 2^{j+1} \mathfrak{m}$ then there is $\beta \in 2 \mathfrak{m}$ such that $1+\alpha=(1+\beta)^{2^{j}}$.

(ii) If $\alpha \in 2^{j+1} \mathcal{O}^{\times}$and the extension $k / \mathbb{Q}_{2}$ is totally ramified then there is $\beta \in 2 \mathfrak{m}$ such that $1+\alpha=5^{2^{j-1}}(1+\beta)^{2^{j}}$.

Proof. We use the following well known result: If $\alpha \in 4 \mathfrak{m}$ then $1+\alpha$ is a square in $k$ and $1+\alpha=(1+\beta)^{2}$ for some $\beta$ with $\beta \mathcal{O}=\frac{1}{2} \alpha \mathcal{O}$. More precisely, $\beta \in \frac{1}{2} \alpha(1+\mathfrak{m})$. One way to prove this is to show that there is a sequence $\beta_{0}, \beta_{1}, \ldots$ with $\beta_{0}=\frac{1}{2} \alpha$ such that $\left(1+\beta_{n}\right)^{2} \equiv 1+\alpha\left(\bmod \alpha \mathfrak{m}^{n+1}\right)$ and $\beta_{n} \equiv \beta_{n+1}\left(\bmod \frac{1}{2} \alpha \mathfrak{m}^{n+1}\right)$ and then take $\beta=\lim \beta_{n}$.

We use induction on $j$. Take $j=1$. For (i) we have $\alpha \in 4 \mathfrak{m}$ and, by the result above, $1+\alpha=(1+\beta)^{2}$ for some $\beta$ such that $\beta \mathcal{O}=\frac{1}{2} \alpha \mathcal{O}$. But $\alpha \in 4 \mathfrak{m}$ so $\beta \in 2 \mathfrak{m}$. For (ii) we have $\alpha \in 4 \mathcal{O}^{\times}$so $\alpha=4 \eta$ for some $\eta \in \mathcal{O}^{\times}$. We now use the fact that $k / \mathbb{Q}_{2}$ is a totally ramified extension so its inertia degree $[\mathcal{O} / \mathfrak{m}: \mathbb{Z} / 2 \mathbb{Z}]$ is 1 . Thus $\mathcal{O} / \mathfrak{m} \cong \mathbb{Z} / 2 \mathbb{Z}=\{\widehat{0}, \widehat{1}\}$. Since $\eta \notin \mathfrak{m}$ we have $\widehat{\eta} \neq \widehat{0}$ so $\widehat{\eta}=\widehat{1}$. It follows that $\eta \equiv 1(\bmod \mathfrak{m})$, which implies $1+\alpha=1+4 \eta \equiv 5$ $(\bmod 4 \mathfrak{m})$ so $5^{-1}(1+\alpha) \equiv 1(\bmod 4 \mathfrak{m})$. Hence $5^{-1}(1+\alpha)=1+\alpha_{1}$ for some $\alpha_{1} \in 4 \mathfrak{m}$. By (i) we have $1+\alpha_{1}=(1+\beta)^{2}$ for some $\beta \in 2 \mathfrak{m}$ so $1+\alpha=5(1+\beta)^{2}$.

Let now $j>1$. We have $\alpha \in 2^{j+1} \mathfrak{m}$ or $2^{j+1} \mathcal{O}^{\times}$. In both cases $\alpha \in$ $2^{j+1} \mathcal{O} \subseteq 4 \mathfrak{m}$. This implies that $1+\alpha=(1+\gamma)^{2}$ for some $\gamma$ such that $\gamma \mathcal{O}=\frac{1}{2} \alpha \mathcal{O}$. In the case of (i) this implies $\gamma \in 2^{j} \mathfrak{m}$, while in the case of (ii), $\gamma \in 2^{j} \mathcal{O}^{\times}$. By the induction hypothesis we have $1+\gamma=(1+\beta)^{2^{j-1}}$ or $5^{2^{j-2}}(1+\beta)^{2^{j-1}}$, respectively, for some $\beta \in 2 \mathfrak{m}$. Since $1+\alpha=(1+\gamma)^{2}$ we get the desired results.

1.7. Suppose now that $p, p^{\prime}$ are two primes $\equiv 3,7(\bmod 20)$ and assume that $p \equiv p^{\prime}(\bmod 16)$ and $y \equiv \pm y^{\prime}(\bmod 8)$.

From $x^{2}+5 y^{2}=u^{2} \pm 2 v^{2}=2 p$ we see that $x, y, v$ are odd and $u$ is even. If $p \equiv 3(\bmod 8)$ then $u^{2}=2 p-2 v^{2} \equiv 2 \cdot 3-2 \cdot 1=4(\bmod 8)$ so $4 \nmid u$.

If $p \equiv 7(\bmod 8)$ then $u^{2}=2 p+2 v^{2} \equiv 2 \cdot 3+2 \cdot 1 \equiv 0(\bmod 8)$ so $4 \mid u$. We reduce to the case when $8 \mid u$. If $u \equiv 4(\bmod 8)$ then we replace $u, v$ with $u_{1}=3 u+4 v$ and $v_{1}=2 u+3 v \cdot(3+2 \sqrt{2}$ is a unit of norm 1 in $\mathbb{Z}[\sqrt{2}]$ and $(u+v \sqrt{2})(3+2 \sqrt{2})=u_{1}+v_{1} \sqrt{2}$. $)$ Since $u \equiv 4(\bmod 8)$ and $v$ is odd we have $8 \mid 3 u+4 v=u_{1}$.

Similarly for $x^{\prime}, y^{\prime}, u^{\prime}, v^{\prime}$.

Since $x, y, v, x^{\prime}, y^{\prime}, v^{\prime}$ are all odd we may assume, after multiplying $x, y, v$ with \pm 1 , that $x, y, v \equiv x^{\prime}, y^{\prime}, v^{\prime}(\bmod 4)$. If $p \equiv p^{\prime} \equiv 3(\bmod 8)$ then $u / 2, u^{\prime} / 2$ are odd, so after multiplying $u$ with \pm 1 , we may assume that $u / 2 \equiv u^{\prime} / 2(\bmod 4)$ so $u \equiv u^{\prime}(\bmod 8)$. The same happens if $p \equiv p^{\prime} \equiv 7$ 
$(\bmod 8)$, when both $u, u^{\prime}$ are multiples of 8. Since $x \equiv x^{\prime}(\bmod 4)$ and $x$ is odd we have $x+x^{\prime} \equiv 2 x \equiv 2(\bmod 4)$, and similarly for $y+y^{\prime}$ and $v+v^{\prime}$.

We have $2 p \equiv 2 p^{\prime}(\bmod 32)$ so $x^{2}+5 y^{2} \equiv x^{\prime 2}+5 y^{\prime 2}(\bmod 32)$ and $u^{2} \pm 2 v^{2} \equiv u^{\prime 2} \pm 2 v^{\prime 2}(\bmod 32)$.

If $p \equiv p^{\prime} \equiv 3(\bmod 8)$ then $u / 2$ and $u^{\prime} / 2$ are odd so $u^{2} / 4 \equiv u^{\prime 2} / 4 \equiv 1$ $(\bmod 8)$. Thus $u^{2} \equiv u^{\prime 2}(\bmod 32)$. The same happens if $p \equiv 7(\bmod 8)$, when $8 \mid u, u^{\prime}$. Together with $u^{2} \pm 2 v^{2} \equiv u^{\prime 2} \pm 2 v^{\prime 2}(\bmod 32)$, this implies $v^{2} \equiv v^{\prime 2}$ $(\bmod 16)$. Since $16 \mid v^{2}-v^{\prime 2}$ and $v+v^{\prime} \equiv 2(\bmod 4)$ we have $8 \mid v-v^{\prime}$ so $v \equiv v^{\prime}(\bmod 8)$.

Since $y \equiv y^{\prime}(\bmod 4)$ and $y \equiv \pm y^{\prime}(\bmod 8)$ we have $y \equiv y^{\prime}(\bmod 8)$. This implies that $y^{2} \equiv y^{\prime 2}(\bmod 16)$. (We have $2 \mid y+y^{\prime}$ and $8 \mid y-y^{\prime}$ so $16 \mid y^{2}-y^{\prime 2}$.) Since $x^{2}+5 y^{2} \equiv x^{\prime 2}+5 y^{\prime 2}(\bmod 32)$ and $y^{2} \equiv y^{\prime 2}(\bmod 16)$ we have $x^{2} \equiv x^{\prime 2}(\bmod 16)$. Thus $16 \mid x^{2}-x^{\prime 2}$ and $x+x^{\prime} \equiv 2(\bmod 4)$ so $8 \mid x-x^{\prime}$. Let $x^{\prime}-x=8 a$ and $y^{\prime}-y=8 b$. Then

$$
\begin{aligned}
x^{2}+5 y^{2} & \equiv x^{\prime 2}+5 y^{\prime 2}=(x+8 a)^{2}+5(y+8 b)^{2} \\
& \equiv x^{2}+16 x a+5 y^{2}+80 y b(\bmod 32)
\end{aligned}
$$

so $2 \mid x a+5 y b$. But $x, y$ are odd so we get $a \equiv b(\bmod 2)$.

In conclusion, we reduced to the case when $x, y, u, v \equiv x^{\prime}, y^{\prime}, u^{\prime}, v^{\prime}(\bmod 8)$, and if $x-x^{\prime}=8 a$ and $y-y^{\prime}=8 b$, then $a \equiv b(\bmod 2)$. Also $x, y, v, x^{\prime}, y^{\prime}, v^{\prime}$ are odd, and if $p \equiv p^{\prime} \equiv 3(\bmod 8)$, then $u / 2, u^{\prime} / 2$ are odd as well.

Note that 2 is inert in $F=\mathbb{Q}(\sqrt{5})$ and is totally ramified in $\mathbb{Q}(\zeta)=$ $\mathbb{Q}(i, \sqrt{2})$, so in $E=F(\zeta)=\mathbb{Q}(\sqrt{5}, i, \sqrt{2})$ there is only one prime $\mathfrak{Q}$ over 2 with ramification index $e_{\mathfrak{Q} / 2}=4$. Let $\mathfrak{q}_{1}, \mathfrak{q}_{2}$ be the primes of $\mathbb{Q}(\sqrt{5} i)$ and $\mathbb{Q}(\sqrt{2} i)($ if $p \equiv 3(\bmod 8))$ or $\mathbb{Q}(\sqrt{2})($ if $p \equiv 7(\bmod 8))$ lying over 2 . We have $e_{\mathfrak{q}_{1} / 2}=e_{\mathfrak{q}_{2} / 2}=2$ so $e_{\mathfrak{Q} / \mathfrak{q}_{1}}=e_{\mathfrak{Q} / \mathfrak{q}_{2}}=2$.

Denote by $\mathcal{O}_{\mathfrak{q}}$ the ring of integers in $F_{\mathfrak{q}}$ and by $\widetilde{\mathfrak{q}}=\mathfrak{q} \mathcal{O}_{\mathfrak{q}}$ the maximal ideal of $\mathcal{O}_{\mathfrak{q}}$. We do the same for $\mathfrak{q}_{1}, \mathfrak{q}_{2}, \mathfrak{Q}$. Also denote by $\mathcal{O}_{2}$ the ring of integers in $\mathbb{Q}_{2}$ and by $\widetilde{2}=2 \mathcal{O}_{2}$ the maximal ideal of $\mathcal{O}_{2}$.

Lemma 1.8. If $A_{1}^{\prime}$ is the $A_{1}$ corresponding to $p^{\prime}$ then $A_{1}^{\prime}=\left(\sqrt{5}^{s} t\right)^{8} A_{1}$ for some integer $s$ and some $t \in 1+2 \widetilde{\mathfrak{Q}}$.

Proof. If $p \equiv 3(\bmod 8)$ then $\widetilde{\mathfrak{q}}_{2}$ is generated by $\sqrt{2} i$. Since $u$ is even and $v$ is odd we have $\operatorname{ord}_{\widetilde{\mathfrak{q}}_{2}}(u+v \sqrt{2} i)=1$. Now $8 \mid u^{\prime}-u$ and $8 \mid v^{\prime}-v$ so $\left(u^{\prime}+v^{\prime} \sqrt{2} i\right)-(u+v \sqrt{2} i)=\left(u^{\prime}-u\right)+\left(v^{\prime}-v\right) \sqrt{2} i \in 8 \mathcal{O}_{\mathfrak{q}_{2}}$. Together with $\operatorname{ord}_{\widetilde{\mathfrak{q}}_{2}}(u+v \sqrt{2} i)=1$, this implies that

$$
\frac{u^{\prime}+v^{\prime} \sqrt{2} i}{u+v \sqrt{2} i}-1 \in 8 \widetilde{\mathfrak{q}}_{2}^{-1}=4 \widetilde{\mathfrak{q}}_{2} .
$$

By Lemma 1.6(i) applied to $k=\mathbb{Q}(\sqrt{2} i)_{\mathfrak{q}_{2}}$, we get 


$$
\frac{u^{\prime}+v^{\prime} \sqrt{2} i}{u+v \sqrt{2} i}=t_{1}^{2} \quad \text { with } t_{1} \in 1+2 \widetilde{\mathfrak{q}}_{2} \subset 1+2 \widetilde{\mathfrak{Q}} .
$$

Similarly for $\left(u^{\prime}+v^{\prime} \sqrt{2}\right) /(u+v \sqrt{2})$ when $p \equiv 7(\bmod 8)$.

We have $\mathrm{N}_{\mathbb{Q}(\sqrt{5} i)_{\mathfrak{q}_{1}} / \mathbb{Q}_{2}}(x+y \sqrt{5} i)=2 p$ so ord $\tilde{\mathfrak{q}}_{1}(x+y \sqrt{5} i)=\frac{1}{2} \operatorname{ord}_{\tilde{\mathfrak{q}}_{1}} 2 p=1$. Now $\left(x^{\prime}+y^{\prime} \sqrt{5} i\right)-(x+y \sqrt{5} i)=8 a+8 b \sqrt{5} i$, where $a, b \in \mathbb{Z}$ have the same parity. But $\mathrm{N}_{\mathbb{Q}(\sqrt{5} i)_{\mathfrak{q}_{1} / \mathbb{Q}_{2}}}(a+b \sqrt{5} i)=a^{2}+5 b^{2}$ is even so $a+b \sqrt{5} i \in \widetilde{\mathfrak{q}}_{1}$. It follows that $\left(x^{\prime}+y^{\prime} \sqrt{5} i\right)-(x+y \sqrt{5} i) \in 8 \widetilde{\mathfrak{q}}_{1}$, which, together with $\operatorname{ord}_{\widetilde{\mathfrak{q}}_{1}}(x+$ $y \sqrt{5} i)=1$, implies that

$$
\frac{x^{\prime}+y^{\prime} \sqrt{5} i}{x+y \sqrt{5} i}-1 \in 8 \mathcal{O}_{\mathfrak{q}_{1}} .
$$

By Lemma $1.6(\mathrm{i})$ and (ii) applied to $k=\mathbb{Q}(\sqrt{5} i)_{\mathfrak{q}_{1}}$, this implies that

$$
\frac{x^{\prime}+y^{\prime} \sqrt{5} i}{x+y \sqrt{5} i}=5^{2 s_{2}} t_{2}^{4}, \quad \text { where } \quad s_{2} \in\{0,1\}, t_{2} \in 1+2 \widetilde{\mathfrak{q}}_{1} \subset 1+2 \widetilde{\mathfrak{Q}} .
$$

Since $p \equiv p^{\prime}(\bmod 16)$ we have $p^{\prime} / p \in 1+16 \mathcal{O}_{2}$. By Lemma 1.6(i) and (ii) applied to $k=\mathbb{Q}_{2}$, we have $p^{\prime} / p=5^{4 s_{3}} t_{3}^{8}$ where $s_{3} \in\{0,1\}$ and $t_{3} \in 1+2 \widetilde{2} \subset 1+2 \widetilde{\mathfrak{Q}}$.

If $p \equiv 3(\bmod 8)$ then

$$
A_{1}^{\prime} / A_{1}=5^{4 s_{3}} t_{3}^{8}\left(5^{2 s_{2}} t_{2}^{4}\right)^{2}\left(t_{1}^{2}\right)^{4}=\left(\sqrt{5}^{s} t\right)^{8}
$$

with $s=s_{3}+s_{2}$ and $t=t_{3} t_{2} t_{1}$. If $p \equiv 7(\bmod 8)$ then

$$
A_{1}^{\prime} / A_{1}=5^{4 s_{3}} t_{3}^{8}\left(5^{2 s_{2}} t_{2}^{4}\right)^{6}\left(t_{1}^{2}\right)^{4}=\left(\sqrt{5}^{s} t\right)^{8}
$$

with $s=s_{3}+3 s_{2}$ and $t=t_{3} t_{2}^{3} t_{1}$. Since $t_{1}, t_{2}, t_{3}$ belong to $1+2 \widetilde{\mathfrak{Q}}$, so does $t$.

LEMMA 1.9. We have

$$
\chi\left(\left(\frac{\varepsilon, L / F}{\mathfrak{q}}\right)\right)=\chi^{\prime}\left(\left(\frac{\varepsilon, L^{\prime} / F}{\mathfrak{q}}\right)\right) .
$$

Proof. Let $\phi=\left(\frac{\varepsilon, L / F}{\mathfrak{q}}\right)$. Then $\phi=\left(\varepsilon, L_{\mathcal{Q}} / F_{\mathfrak{q}}\right)$, where $\mathcal{Q}$ is a prime of $L$ over $\mathfrak{Q}$, so over $\mathfrak{q}$. Since $-1,2 \in \mathbb{Q}_{2}$ and $\mathrm{N}_{\mathbb{Q}(\sqrt{5})_{\mathfrak{q}} / \mathbb{Q}_{2}}(\varepsilon)=-1$ we have

$$
\left(\frac{\varepsilon,-1}{\mathfrak{q}}\right)=\left(\frac{-1,-1}{2}\right)=-1 \quad \text { and } \quad\left(\frac{\varepsilon, 2}{\mathfrak{q}}\right)=\left(\frac{-1,2}{2}\right)=1
$$

so $\phi(i)=-i$ and $\phi(\sqrt{2})=\sqrt{2}$. It follows that $\phi(\zeta)=\bar{\zeta}=\zeta^{-1}=\zeta^{7}=\tau_{7}(\zeta)$. So $\phi_{\mid E}=\tau_{7 \mid E}$, which implies that $\phi \in \tau_{7} \operatorname{Gal}(L / E)=\tau_{7}\langle\sigma\rangle$. So $\phi=\sigma^{k} \tau_{7}$ for some $k \in \mathbb{Z}_{8}$. We have $\chi(\phi)=\chi\left(\sigma^{k} \tau_{7}\right)=\zeta^{k}$.

We have $\tau_{7}\left(\alpha_{1}\right)=\alpha_{7}=2 p \alpha_{1}^{-1}$ or $2 p(u+v \sqrt{2}) \alpha_{1}^{-1}$, corresponding to $p \equiv 3$ or $7(\bmod 8)$. So $\phi\left(\alpha_{1}\right)=\sigma^{k} \tau_{7}\left(\alpha_{1}\right)=\sigma^{k}\left(2 p \alpha_{1}^{-1}\right)=2 p \zeta^{-k} \alpha_{1}^{-1}$ or $\phi\left(\alpha_{1}\right)=2 p(u+v \sqrt{2}) \zeta^{-k} \alpha_{1}^{-1}$, respectively. 
We make the same reasoning for $p^{\prime}$. Denote by $\phi^{\prime}, \mathcal{Q}^{\prime}, k^{\prime}$ the $\phi, \mathcal{Q}, k$ corresponding to $p^{\prime}$. Our goal is to prove that $\zeta^{k}=\zeta^{k^{\prime}}$.

We have $L_{\mathcal{Q}}=E_{\mathfrak{Q}}\left(\alpha_{1}\right), \alpha_{1}^{8}=A_{1} \in E_{\mathfrak{Q}}$, and $\mu_{8} \subset E_{\mathfrak{Q}}$. It follows that $L_{\mathcal{Q}}$ is the splitting field of $X^{8}-A_{1} \in E_{\mathfrak{Q}}[X]$. Similarly $L_{\mathcal{Q}^{\prime}}^{\prime}$ is the splitting field of $X^{8}-A_{1}^{\prime}$. By Lemma 1.8 we have $A_{1}^{\prime}=B^{8} A_{1}$, where $B=\sqrt{5}^{s} t \in E_{\mathfrak{Q}}$, so $L_{\mathcal{Q}^{\prime}}^{\prime} \cong L_{\mathcal{Q}}$. Let $\psi: L_{\mathcal{Q}^{\prime}}^{\prime} \rightarrow L_{\mathcal{Q}}$ be an isomorphism with $\psi_{\mid E_{\mathfrak{Q}}}=1_{E_{\mathfrak{Q}}}$. We have $\left(\psi\left(\alpha_{1}^{\prime}\right)\right)^{8}=\psi\left(A_{1}^{\prime}\right)=A_{1}^{\prime}=B^{8} A_{1}=\left(B \alpha_{1}\right)^{8}$ so $\psi\left(\alpha_{1}^{\prime}\right)=\zeta^{l} B \alpha_{1}$ for some integer $l$.

Now $\phi=\left(\varepsilon, \psi\left(L_{\mathcal{Q}^{\prime}}^{\prime}\right) / E_{\mathfrak{Q}}\right)=\psi\left(\varepsilon, L_{\mathcal{Q}^{\prime}}^{\prime} / E_{\mathfrak{Q}}\right) \psi^{-1}=\psi \phi^{\prime} \psi^{-1}$ so $\phi \psi=\psi^{\prime} \phi$. In particular, $\phi\left(\psi\left(\alpha_{1}^{\prime}\right)\right)=\psi\left(\phi^{\prime}\left(\alpha_{1}^{\prime}\right)\right)$. But if $p \equiv 3(\bmod 8)$ then

$$
\begin{aligned}
\phi\left(\psi\left(\alpha_{1}^{\prime}\right)\right) & =\phi\left(\zeta^{l} B \alpha_{1}\right)=\zeta^{-l} \phi(B) \cdot 2 p \zeta^{-k} \alpha_{1}^{-1}, \\
\psi\left(\phi^{\prime}\left(\alpha_{1}^{\prime}\right)\right) & =\psi\left(2 p^{\prime} \zeta^{-k^{\prime}} \alpha_{1}^{\prime-1}\right)=2 p^{\prime} \zeta^{-k^{\prime}} \zeta^{-l} B^{-1} \alpha_{1}^{-1} .
\end{aligned}
$$

It follows that $\zeta^{k^{\prime}-k}=\frac{p^{\prime}}{p}(B \phi(B))^{-1}$. We have $B=\sqrt{5}^{s} t$ so $B \phi(B)=$ $5^{s} t \phi(t)$. By Lemma 1.8, $t$ belongs to $1+2 \widetilde{\mathfrak{Q}}$ and so does its conjugate, $\phi(t)$. Since also $5 \in 1+4 \mathcal{O}_{2} \subset 1+2 \widetilde{\mathfrak{Q}}$ we get $B \phi(B) \in 1+2 \widetilde{\mathfrak{Q}}$, which, together with $p^{\prime} / p \in 1+16 \mathcal{O}_{2} \subset 1+2 \widetilde{\mathfrak{Q}}$, implies $\zeta^{k^{\prime}-k} \in 1+2 \widetilde{\mathfrak{Q}}$. By a similar reasoning, if $p \equiv 7(\bmod 8)$ we have

$$
\zeta^{k^{\prime}-k}=\frac{p^{\prime}}{p} \cdot \frac{u^{\prime}+v^{\prime} \sqrt{2}}{u+v \sqrt{2}}(B \phi(B))^{-1} .
$$

Since

$$
\frac{u^{\prime}+v^{\prime} \sqrt{2}}{u+v \sqrt{2}} \in 1+4 \widetilde{\mathfrak{q}}_{2} \subset 1+2 \widetilde{\mathfrak{Q}}
$$

(see the proof of Lemma 1.8) we get again $\zeta^{k^{\prime}-k} \in 1+2 \widetilde{\mathfrak{Q}}$.

We have $\zeta^{k^{\prime}-k} \in 1+2 \widetilde{\mathfrak{Q}}$ so $1-\zeta^{k^{\prime}-k} \in 2 \widetilde{\mathfrak{Q}}$, which implies $\zeta^{k^{\prime}-k}=1$. (If $\eta \in \mu_{8}, \eta \neq 1$, then $1-\eta \mid 2$ so $1-\eta \notin 2 \widetilde{\mathfrak{Q}}$.) Thus $\zeta^{k^{\prime}}=\zeta^{k}$.

Lemma 1.10.

(i) If $p \equiv 3(\bmod 8)$ then

$$
\begin{aligned}
\left(\frac{x}{p}\right) & =\left(\frac{x, 14}{2}\right)\left(\frac{2 p}{5}\right)_{4}, & & \left(\frac{y}{p}\right)=\left(\frac{y, 6}{2}\right), \\
\left(\frac{u}{p}\right) & =\left(\frac{u, 3}{2}\right), & \left(\frac{v}{p}\right) & =\left(\frac{v, 6}{2}\right) .
\end{aligned}
$$

(ii) If $p \equiv 7(\bmod 8)$ then

$$
\begin{aligned}
& \left(\frac{x}{p}\right)=\left(\frac{x, 6}{2}\right)\left(\frac{2 p}{5}\right)_{4}, \quad\left(\frac{y}{p}\right)=\left(\frac{y, 14}{2}\right), \\
& \left(\frac{u}{p}\right)=\operatorname{sgn}(u), \quad\left(\frac{v}{p}\right)=\left(\frac{v, 14}{2}\right) \text {. }
\end{aligned}
$$


Proof. Note that $p \nmid x y u v$.

Let $q \neq 2,5, p$ be a prime. If $q \mid x$, then $2 p=x^{2}+5 y^{2} \equiv 5 y^{2}(\bmod q)$. Hence $\left(\frac{10 p}{q}\right)=1$, which implies $\left(\frac{x, 10 p}{q}\right)=1$. The same happens if $q \nmid x$, when both $x$ and $10 p$ are units in $\mathbb{Q}_{q}$. We also have $10 p>0$ so $\left(\frac{x, 10 p}{\infty}\right)=1$. By Hilbert's reciprocity law we get

$$
\left(\frac{x}{p}\right)=\left(\frac{x, 10 p}{p}\right)=\left(\frac{x, 10 p}{2}\right)\left(\frac{x, 10 p}{5}\right) .
$$

But $5 \nmid x$ so $\left(\frac{x, 10 p}{5}\right)=\left(\frac{x}{5}\right)$. Since $2 p=x^{2}+5 y^{2} \equiv x^{2}(\bmod 5)$ and $\left(\frac{-1}{5}\right)=1$, the quartic residue symbol $\left(\frac{2 p}{5}\right)_{4}$ is defined and is equal to $\left(\frac{x}{5}\right)$. Hence

$$
\left(\frac{x}{p}\right)=\left(\frac{x, 10 p}{2}\right)\left(\frac{2 p}{5}\right)_{4}
$$

But if $p \equiv 3$ or $7(\bmod 8)$ then modulo $\mathbb{Q}_{2}^{\times 2}$ we have $10 p=14$ or 6 , respectively. This yields the formulas for $\left(\frac{x}{p}\right)$ in (i) and (ii).

Similarly if $q \neq 2, p$ is a prime then either $q \mid y$ so $2 p=x^{2}+5 y^{2} \equiv x^{2}$ $(\bmod q)$ so $\left(\frac{2 p}{q}\right)=1$ so $\left(\frac{y, 2 p}{q}\right)=1$, or $q \nmid y$ so again $\left(\frac{y, 2 p}{q}\right)=1$. Also $2 p>0$ so $\left(\frac{y, 2 p}{\infty}\right)=1$. Hence $\left(\frac{y}{p}\right)=\left(\frac{y, 2 p}{p}\right)=\left(\frac{y, 2 p}{2}\right)$. By the same proof $\left(\frac{v}{p}\right)=\left(\frac{v, 2 p}{2}\right)$ (in both cases when $\left.u^{2} \pm 2 v^{2}=2 p\right)$. But if $p \equiv 3$ or $7(\bmod 8)$ then $2 p=6$ or 14 , respectively, in $\mathbb{Q}_{2}^{\times} / \mathbb{Q}_{2}^{\times 2}$. This gives the formulas for $\left(\frac{y}{p}\right)$ and $\left(\frac{v}{p}\right)$ in (i) and (ii).

If $p \equiv 3(\bmod 8)$ then for any prime $q \neq 2, p$ we have either $q \mid u$ so $2 p \equiv u^{2}+2 v^{2} \equiv 2 v^{2}(\bmod p)$ and so $\left(\frac{p}{q}\right)=1$ so $\left(\frac{u, p}{q}\right)=1$, or $q \nmid u$ and again $\left(\frac{u, p}{q}\right)=1$. Similarly if $p \equiv 7(\bmod 8)$ then $2 p=u^{2}-2 v^{2}$ implies $\left(\frac{u,-p}{q}\right)=1$ for $q \neq 2, p$ prime. If $p \equiv 3(\bmod 8)$ then $p>0$ so $\left(\frac{u, p}{\infty}\right)=1$ and so

$$
\left(\frac{u}{p}\right)=\left(\frac{u, p}{p}\right)=\left(\frac{u, p}{2}\right)=\left(\frac{u, 3}{2}\right) \text {. }
$$

If $p \equiv 7(\bmod 8)$ then $-p \in \mathbb{Q}_{2}^{\times 2}$ so $\left(\frac{u,-p}{2}\right)=1$. We get

$$
\left(\frac{u}{p}\right)=\left(\frac{u,-p}{p}\right)=\left(\frac{u,-p}{\infty}\right)=\operatorname{sgn}(u)
$$

REMARK 1.11. If $s, t$ are $p$-adic units and $s \equiv \pm t(\bmod p)$ then

$$
s \equiv\left(\frac{s t}{p}\right) t(\bmod p) .
$$

Indeed, if $s \equiv t(\bmod p)$ then $\left(\frac{s t}{p}\right)=1$, while if $s \equiv-t(\bmod p)$ then $\left(\frac{s t}{p}\right)=\left(\frac{-1}{p}\right)=-1$. Also note that, since $\left(\frac{-1}{p}\right)=-1$, we have $\left(\frac{\alpha}{p}\right)=\alpha$ if $\alpha \in\{ \pm 1\}$. 
Lemma 1.12. Let $\alpha, \beta \in\{ \pm 1\}$ and let $s, t$ be integers in $\mathbb{Q}_{p}$ such that $s+t \sqrt{5} \equiv \frac{\alpha+\beta i}{\sqrt{2}}(\bmod \widetilde{\mathfrak{P}})$.

(i) If $p \equiv 3(\bmod 8)$ then

$$
\begin{aligned}
s & \equiv-2^{(p-3) / 4}\left(\frac{u, 3}{2}\right)\left(\frac{v, 6}{2}\right) \beta(\bmod p), \\
t & \equiv-10^{(p-3) / 4}\left(\frac{x, 14}{2}\right)\left(\frac{y, 6}{2}\right)\left(\frac{u, 3}{2}\right)\left(\frac{v, 6}{2}\right)\left(\frac{2 p}{5}\right)_{4} \alpha(\bmod p) .
\end{aligned}
$$

(ii) If $p \equiv 7(\bmod 8)$ then

$$
\begin{aligned}
s & \equiv 2^{(p-3) / 4}\left(\frac{v, 14}{2}\right) \operatorname{sgn}(u) \alpha(\bmod p), \\
t & \equiv 10^{(p-3) / 4}\left(\frac{x, 6}{2}\right)\left(\frac{y, 14}{2}\right)\left(\frac{v, 14}{2}\right)\left(\frac{2 p}{5}\right)_{4} \operatorname{sgn}(u) \beta(\bmod p) .
\end{aligned}
$$

Proof. (i) We have $p \nmid x y u v$ and $x-y \sqrt{5} i, u-v \sqrt{2} i \in \widetilde{\mathfrak{P}}$ so $x \equiv y \sqrt{5} i$ $(\bmod \widetilde{\mathfrak{P}})$ and $u \equiv v \sqrt{2} i(\bmod \widetilde{\mathfrak{P}})$. It follows that

$$
\frac{i}{\sqrt{2}} \equiv-\frac{v}{u}(\bmod \widetilde{\mathfrak{P}}), \quad \frac{1}{\sqrt{2}} \equiv-\frac{y v}{x u} \sqrt{5}(\bmod \widetilde{\mathfrak{P}}) .
$$

Hence

$$
s+t \sqrt{5} \equiv \frac{\alpha+\beta i}{\sqrt{2}} \equiv-\alpha \frac{y v}{x u} \sqrt{5}-\beta \frac{v}{u}(\bmod \widetilde{\mathfrak{P}}) .
$$

Since both sides belong to $F_{\mathfrak{p}}$, the congruence will also hold modulo $\widetilde{\mathfrak{p}}$. This implies

$$
s \equiv-\beta \frac{v}{u}(\bmod p), \quad t \equiv-\alpha \frac{y v}{x u}(\bmod p) .
$$

We have

$$
s \equiv-\beta \frac{v}{u} \equiv \frac{\beta i}{\sqrt{2}}(\bmod \widetilde{\mathfrak{P}}), \quad t \sqrt{5} \equiv-\alpha \frac{y v}{x u} \sqrt{5} \equiv \frac{\alpha}{\sqrt{2}}(\bmod \widetilde{\mathfrak{P}}) .
$$

Taking squares yields $s^{2} \equiv-\frac{1}{2}(\bmod \widetilde{\mathfrak{P}})$ and $5 t^{2} \equiv \frac{1}{2}(\bmod \widetilde{\mathfrak{P}})$ so $t^{2} \equiv \frac{1}{10}$ $(\bmod \widetilde{\mathfrak{P}})$. Since all sides belong to $\mathbb{Q}_{p}$, these congruences will also hold modulo $p$. It follows that $\left(\frac{-2}{p}\right)=\left(\frac{10}{p}\right)=1$ and so $(-2)^{(p-1) / 2} \equiv 10^{(p-1) / 2} \equiv 1$ $(\bmod p)$. Thus $s^{2} \equiv \frac{1}{-2} \equiv\left((-2)^{(p-3) / 4}\right)^{2}(\bmod p)$ so $s \equiv \pm(-2)^{(p-3) / 4}$ and similarly $t \equiv \pm 10^{(p-3) / 4}(\bmod p)$.

By Lemma 1.10 and Remark 1.11 we have

$$
\begin{aligned}
s & \equiv\left(\frac{(-2)^{(p-3) / 4} s}{p}\right)(-2)^{(p-3) / 4}=\left(\frac{s}{p}\right) 2^{(p-3) / 4} \\
& =\left(\frac{-u v \beta}{p}\right) 2^{(p-3) / 4}=-2^{(p-3) / 4}\left(\frac{u, 3}{2}\right)\left(\frac{v, 6}{2}\right) \beta(\bmod p) .
\end{aligned}
$$


(Note that $(p-3) / 4$ is even.) Similarly

$$
\begin{aligned}
t & \equiv\left(\frac{10^{(p-3) / 4} t}{p}\right) 10^{(p-3) / 4}=\left(\frac{t}{p}\right) 10^{(p-3) / 4}=\left(\frac{-x y u v \alpha}{p}\right) 10^{(p-3) / 4} \\
& =-10^{(p-3) / 4}\left(\frac{x, 14}{2}\right)\left(\frac{y, 6}{2}\right)\left(\frac{u, 3}{2}\right)\left(\frac{v, 6}{2}\right)\left(\frac{2 p}{5}\right)_{4} \alpha(\bmod p) .
\end{aligned}
$$

If $p \equiv 7(\bmod 8)$ then again $x \equiv y \sqrt{5} i(\bmod \widetilde{\mathfrak{P}})$ but $u \equiv v \sqrt{2}(\bmod \widetilde{\mathfrak{P}})$. So this time

$$
\frac{1}{\sqrt{2}} \equiv \frac{v}{u}(\bmod \tilde{\mathfrak{P}}), \quad \frac{i}{\sqrt{2}} \equiv-\frac{y v}{x u} \sqrt{5}
$$

We get

$$
s+t \sqrt{5} \equiv \frac{v}{u} \alpha-\frac{y v}{x u} \sqrt{5}(\bmod \widetilde{\mathfrak{P}})
$$

so

$$
s \equiv \frac{v}{u} \alpha(\bmod p) \quad \text { and } \quad t \equiv-\frac{y v}{x u} \beta(\bmod p) .
$$

Now

$$
s \equiv \alpha \frac{v}{u} \equiv \frac{\alpha}{\sqrt{2}}(\bmod \widetilde{\mathfrak{P}}), \quad t \sqrt{5} \equiv-\beta \frac{y v}{x u} \sqrt{5} \equiv \frac{\beta i}{\sqrt{2}}(\bmod \widetilde{\mathfrak{P}}) .
$$

Just as for the case when $p \equiv 3(\bmod 8)$, we get $s^{2} \equiv \frac{1}{2}(\bmod p)$ and $5 t^{2} \equiv-\frac{1}{2}(\bmod p)$ and so $t^{2} \equiv-\frac{1}{10}(\bmod p)$, which, by the same argument, will imply

$$
s \equiv\left(\frac{s}{p}\right) 2^{(p-3) / 4} \equiv\left(\frac{u v \alpha}{p}\right)=\left(\frac{v, 14}{2}\right) \operatorname{sgn}(u) \alpha(\bmod p)
$$

and

$$
\begin{aligned}
t & \equiv\left(\frac{t}{p}\right)(-10)^{(p-3) / 4} \equiv-\left(\frac{-x y u v \beta}{p}\right) 10^{(p-3) / 4} \\
& =10^{(p-3) / 4}\left(\frac{x, 6}{2}\right)\left(\frac{y, 14}{2}\right)\left(\frac{v, 14}{2}\right)\left(\frac{2 p}{5}\right)_{4} \operatorname{sgn}(u) \beta(\bmod p),
\end{aligned}
$$

as claimed. (Note that this time $(p-3) / 4$ is odd so $(-10)^{(p-3) / 4}=$ $-10^{(p-3) / 4}$.)

LEMma 1.13. We have $F_{(p+1) / 4} \equiv 2 \cdot 10^{(p-3) / 4}\left(\frac{2 p}{5}\right)_{4} A(\bmod p), \quad L_{(p+1) / 4} \equiv 2^{(p+1) / 4} B(\bmod p)$, where $A, B \in\{ \pm 1\}$ depend only on $p \bmod 16$ and $\pm y \bmod 8$.

Proof. As seen in $1.2, \varepsilon^{p+1} \equiv-1(\bmod \mathfrak{p})$. This congruence also holds modulo $\mathfrak{P}$ so $\varepsilon^{(p+1) / 4} \equiv \eta(\bmod \mathfrak{P})$ for some primitive $\eta \in \mu_{8}$. 
By 1.2 we have

$$
\varepsilon^{(p+1) / 4} \equiv \begin{cases}(-1)^{(p-3) / 8} \varepsilon^{\left(p^{2}-1\right) / 8}(\bmod p) & \text { if } p \equiv 3(\bmod 8), \\ (-1)^{(p+1) / 8} \varepsilon^{-\left(p^{2}-1\right) / 8}(\bmod p) & \text { if } p \equiv 7(\bmod 8) .\end{cases}
$$

These congruences also hold modulo $\mathfrak{P}$. Together with 1.5 they imply that $\varepsilon^{(p+1) / 4} \equiv \nu(\bmod \mathfrak{P})$, where $\nu \in \mu_{8}$ is given by

$$
\nu= \begin{cases}(-1)^{(p-3) / 8} \chi\left(\left(\frac{\varepsilon, L / F}{\mathfrak{q}}\right)\right) & \text { if } p \equiv 3(\bmod 8), \\ (-1)^{(p+1) / 8} \operatorname{sgn}(u) \chi\left(\left(\frac{\varepsilon, L / F}{\mathfrak{q}}\right)\right)^{-1} & \text { if } p \equiv 7(\bmod 8) .\end{cases}
$$

It follows that $\eta \equiv \nu(\bmod \mathfrak{P})$, which implies that $\eta=\nu$. (If $\eta_{1}, \eta_{2} \in \mu_{8}$ and $\eta_{1} \equiv \eta_{2}(\bmod \mathfrak{P})$ then $\eta_{1}=\eta_{2}$ since otherwise $\eta_{1}-\eta_{2} \mid 2$ so $\eta_{1}-\eta_{2} \notin \mathfrak{P}$.) In particular, since $\eta=\nu$ is primitive in $\mu_{8}$, so is $\chi\left(\left(\frac{\varepsilon, L / F}{\mathfrak{q}}\right)\right)$.

Let $\chi\left(\left(\frac{\varepsilon, L / F}{\mathfrak{q}}\right)\right)=(\alpha+\beta i) / \sqrt{2}$. We have

where

$$
\frac{L_{(p+1) / 4}}{2}+\frac{F_{(p+1) / 4}}{2} \sqrt{5}=\varepsilon^{(p+1) / 4} \equiv \eta(\bmod \tilde{\mathfrak{P}}),
$$

and

$$
\eta=(-1)^{(p-3) / 8} \frac{\alpha+\beta i}{\sqrt{2}} \quad \text { if } p \equiv 3(\bmod 8)
$$

$$
\eta=(-1)^{(p+1) / 8} \operatorname{sgn}(u)\left(\frac{\alpha+\beta i}{\sqrt{2}}\right)^{-1}=(-1)^{(p+1) / 8} \operatorname{sgn}(u)\left(\frac{\alpha-\beta i}{\sqrt{2}}\right)
$$

if $p \equiv 7(\bmod 8)$. By Lemma 1.12 this implies that

$$
\frac{F_{(p+1) / 4}}{2} \equiv 10^{(p-3) / 4}\left(\frac{2 p}{5}\right)_{4} A(\bmod p), \quad \frac{L_{(p+1) / 4}}{2} \equiv 2^{(p-3) / 4} B(\bmod p),
$$

where $A, B \in\{ \pm 1\}$ are given by

$$
A= \begin{cases}-(-1)^{(p-3) / 8}\left(\frac{x, 14}{2}\right)\left(\frac{y, 6}{2}\right)\left(\frac{u, 3}{2}\right)\left(\frac{v, 6}{2}\right) \alpha & \text { if } p \equiv 3(\bmod 8), \\ -(-1)^{(p+1) / 8}\left(\frac{x, 6}{2}\right)\left(\frac{y, 14}{2}\right)\left(\frac{v, 14}{2}\right) \beta & \text { if } p \equiv 7(\bmod 8),\end{cases}
$$

and

$$
B= \begin{cases}-(-1)^{(p-3) / 8}\left(\frac{u, 3}{2}\right)\left(\frac{v, 6}{2}\right) \beta & \text { if } p \equiv 3(\bmod 8), \\ (-1)^{(p+1) / 8}\left(\frac{v, 14}{2}\right) \alpha & \text { if } p \equiv 7(\bmod 8) .\end{cases}
$$

We still have to prove that $A, B$ depend only on $p \bmod 16$ and $\pm y \bmod 8$. Suppose that $p \equiv p^{\prime}(\bmod 16)$ and $y \equiv \pm y^{\prime}(\bmod 8)$. Denote by $\alpha^{\prime}, \beta^{\prime}, A^{\prime}, B^{\prime}$ the $\alpha, \beta, A, B$ corresponding to $p^{\prime}$. We have to prove that $A=A^{\prime}$ and 
$B=B^{\prime}$. By 1.7 we can restrict ourselves to the case when $x, y, u, v \equiv$ $x^{\prime}, y^{\prime}, u^{\prime}, v^{\prime}(\bmod 8)$. Also $x, y, v, x^{\prime}, y^{\prime}, v^{\prime}$ are odd and, if $p \equiv 3(\bmod 8)$, then $u / 2, u^{\prime} / 2$ are odd integers. This implies that $x x^{\prime}, y y^{\prime}, v v^{\prime} \in \mathbb{Q}_{2}^{\times 2}$. Moreover, if $p \equiv 3(\bmod 8)$, then $u / 2, u^{\prime} / 2$ are odd and $u / 2 \equiv u^{\prime} / 2(\bmod 4)$ so $u u^{\prime} \in \mathbb{Q}_{2}^{\times 2} \cup 5 \mathbb{Q}_{2}^{\times 2}$.

To prove that $A=A^{\prime}$ we show that the various factors that occur in $A$ are equal to the similar factors from $A^{\prime}$, and similarly for $B=B^{\prime}$. By Lemma 1.9 we have $\chi\left(\left(\frac{\varepsilon, L / F}{\mathfrak{q}}\right)\right)=\chi^{\prime}\left(\left(\frac{\varepsilon, L^{\prime} / F}{\mathfrak{q}}\right)\right)$ so $\alpha=\alpha^{\prime}$ and $\beta=\beta^{\prime}$.

If $p \equiv p^{\prime} \equiv 3(\bmod 8)$ then $p \equiv p^{\prime}(\bmod 16)$ implies that $(-1)^{(p-3) / 8}=$ $(-1)^{\left(p^{\prime}-3\right) / 8}$. From $x x^{\prime}, y y^{\prime}, v v^{\prime} \in \mathbb{Q}_{2}^{\times 2}$ and $u u^{\prime} \in \mathbb{Q}_{2}^{\times 2} \cup 5 \mathbb{Q}_{2}^{\times 2}$ we also have

$$
\left(\frac{x x^{\prime}, 14}{2}\right)=\left(\frac{y y^{\prime}, 6}{2}\right)=\left(\frac{u u^{\prime}, 3}{2}\right)=\left(\frac{v v^{\prime}, 6}{2}\right)=1
$$

so

$$
\begin{aligned}
\left(\frac{x, 14}{2}\right) & =\left(\frac{x^{\prime}, 14}{2}\right), & \left(\frac{y, 6}{2}\right) & =\left(\frac{y^{\prime}, 6}{2}\right), \\
\left(\frac{u, 3}{2}\right) & =\left(\frac{u^{\prime}, 3}{2}\right), & \left(\frac{v, 6}{2}\right) & =\left(\frac{v^{\prime}, 6}{2}\right) .
\end{aligned}
$$

Together with $\alpha=\alpha^{\prime}$ and $\beta=\beta^{\prime}$, these imply $A=A^{\prime}$ and $B=B^{\prime}$.

If $p \equiv p^{\prime} \equiv 7(\bmod 8)$ then $p \equiv p^{\prime}(\bmod 16)$ implies that $(-1)^{(p-3) / 8}=$ $(-1)^{\left(p^{\prime}-3\right) / 8}$ and $x x^{\prime}, y y^{\prime}, v v^{\prime} \in \mathbb{Q}_{2}^{\times 2}$ implies that

$$
\left(\frac{x x^{\prime}, 6}{2}\right)=\left(\frac{y y^{\prime}, 14}{2}\right)=\left(\frac{v v^{\prime}, 14}{2}\right)=1
$$

so

$$
\left(\frac{x, 6}{2}\right)=\left(\frac{x^{\prime}, 6}{2}\right), \quad\left(\frac{y, 14}{2}\right)=\left(\frac{y^{\prime}, 14}{2}\right), \quad\left(\frac{v, 14}{2}\right)=\left(\frac{v^{\prime}, 14}{2}\right) .
$$

Together with $\alpha=\alpha^{\prime}$ and $\beta=\beta^{\prime}$, these imply $A=A^{\prime}$ and $B=B^{\prime}$.

Proof of Conjecture 1.1. Note that the factor $(-1)^{[(p-5) / 10]}$ which appears in the expression for $F_{(p+1) / 4} \bmod p$ is equal to $-\left(\frac{2 p}{5}\right)_{4}$. (If $p \equiv 3$ $(\bmod 20)$ they are both -1 ; if $p \equiv 7(\bmod 20)$ they are both 1 .) Therefore Sun's conjecture states that $F_{(p+1) / 4} \equiv 2 \cdot 10^{(p-3) / 4}\left(\frac{2 p}{5}\right){ }_{4} A(\bmod p)$ and $L_{(p+1) / 4} \equiv 2^{(p+1) / 4} B(\bmod p)$ where

$$
\begin{aligned}
& A= \begin{cases}-1 & \text { if } y \equiv \pm \frac{p-1}{2}(\bmod 8), \\
1 & \text { if } y \not \equiv \pm \frac{p-1}{2}(\bmod 8),\end{cases} \\
& B= \begin{cases}(-1)^{(p+1) / 4} & \text { if } y \equiv \pm \frac{p-1}{2}(\bmod 8), \\
(-1)^{(p-3) / 4} & \text { if } y \neq \equiv \pm \frac{p-1}{2}(\bmod 8) .\end{cases}
\end{aligned}
$$

Obviously $A, B$ defined this way depend only on $p \bmod 16$ and $\pm y \bmod 8$. In view of Lemma 1.13 the conjecture has to be verified only for a set of 
primes $p$ such that $p$ covers all the possible remainders modulo 16 , namely $3,7,11,15$, and $\pm y$ covers the odd remainders modulo 8 , i.e. \pm 1 and \pm 3 . One can check that $3,7,23,43,47,67,107,127$ cover all eight possibilities. (We have $2 \cdot 3=1^{2}+5 \cdot 1^{2}, 2 \cdot 7=3^{2}+5 \cdot 1^{2}, 2 \cdot 23=1^{2}+5 \cdot 3^{2}$, $2 \cdot 43=9^{2}+5 \cdot 1^{2}, 2 \cdot 47=7^{2}+5 \cdot 3^{2}, 2 \cdot 67=3^{2}+5 \cdot 5^{2}, 2 \cdot 107=13^{2}+5 \cdot 3^{2}$ and $2 \cdot 127=3^{2}+5 \cdot 7^{2}$.) But Sun checked the conjecture for primes up to 3000 , including these.

2. $(2+\sqrt{3})^{(p+1) / 8} \bmod p$ when $p \equiv 7(\bmod 24)$. Let

$$
V_{n}+U_{n} \sqrt{3}=(2+\sqrt{3})^{(p+1) / 4} .
$$

Conjecture 2.1 (Z. H. Sun, 1988). If $p$ is a prime, $p \equiv 7(\bmod 24)$, and $x, y \in \mathbb{Z}$ with $x \equiv 1(\bmod 3)$ such that $x^{2}+3 y^{2}=p$, then

$$
\left(\frac{U_{(p+1) / 8}}{p}\right)=(-1)^{\left((x+4)^{2}-4\right) / 32}, \quad\left(\frac{V_{(p+1) / 8}}{p}\right)=(-1)^{\left(x^{2}-4\right) / 32} .
$$

Note that $x^{2}+3 y^{2}=p \equiv 7(\bmod 8)$ implies that $y$ is odd and $x \equiv 2$ $(\bmod 4)$. Therefore

$$
\begin{gathered}
(-1)^{\left((-x+4)^{2}-4\right) / 32}=-(-1)^{\left((x+4)^{2}-4\right) / 32}, \\
(-1)^{\left((-x)^{2}-4\right) / 32}=(-1)^{\left(x^{2}-4\right) / 32} .
\end{gathered}
$$

Since also $\left(\frac{-x}{3}\right)=-\left(\frac{x}{3}\right)$ we can remove the condition $x \equiv 1(\bmod 3)$, provided that we replace $(-1)^{\left((x+4)^{2}-4\right) / 32}$ by $(-1)^{\left((x+4)^{2}-4\right) / 32}\left(\frac{x}{3}\right)$. (If $x \equiv 2$ (mod 3) then replacing $x$ by $-x$ will not change the outcome of the two formulas.)

By Lemma 2.11 these will yield some formula for $(2+\sqrt{3})^{(p+1) / 8} \bmod p$ :

$$
\begin{aligned}
U_{(p+1) / 8} & \equiv-(-1)^{\left((x+4)^{2}-4\right) / 32} 6^{(p-3) / 4}\left(\frac{x}{3}\right)(\bmod p), \\
V_{(p+1) / 8} & \equiv(-1)^{\left(x^{2}-4\right) / 32} 2^{(p-3) / 4}(\bmod p) .
\end{aligned}
$$

An alternative formula for $(2+\sqrt{3})^{(p+1) / 8} \bmod p$ is provided in [L, Exercise 9.9 , p. 315], but it also involves writing $p$ as $p=c^{2}+6 d^{2}=e^{2}-2 f^{2}$ with $c, d, e, f \in \mathbb{Z}$.

We will also prove a related result regarding $(2+\sqrt{3})^{(p+5) / 8} \bmod p$ when $p \equiv 19(\bmod 24)$.

Theorem 2.2. If $p \equiv 19(\bmod 24)$ is a prime and $p=x^{2}+3 y^{2}$ then

$$
U_{(p+5) / 8} \equiv \begin{cases}(-1)^{(x+p+1) / 8} 6^{(p-3) / 4}\left(\frac{x}{3}\right)(\bmod p) & \text { if } 8 \nmid x, \\ (-1)^{(x+p+5) / 8} 2^{(p-3) / 4}(\bmod p) & \text { if } 8 \mid x,\end{cases}
$$


and

$$
V_{(p+5) / 8} \equiv \begin{cases}(-1)^{(x+p+1) / 8} \cdot 3 \cdot 6^{(p-3) / 4}\left(\frac{x}{3}\right)(\bmod p) & \text { if } 8 \nmid x, \\ (-1)^{(x+p+5) / 8} 2^{(p-3) / 4}(\bmod p) & \text { if } 8 \mid x .\end{cases}
$$

REMARK. Since $x^{2}+3 y^{2}=p \equiv 3(\bmod 8)$ we have $4 \mid x$. If $x \equiv 4(\bmod 8)$ then $(x+p+1) / 8 \in \mathbb{Z}$ so the formulas above in the case $8 \nmid x$ make sense. Moreover, $(x+p+1) / 8$ and $(-x+p+1) / 8$ have opposite parities, which, together with $\left(\frac{-x}{3}\right)=-\left(\frac{x}{3}\right)$, implies $(-1)^{(x+p+1) / 8}\left(\frac{x}{3}\right)=(-1)^{(-x+p+1) / 8}\left(\frac{-x}{3}\right)$. So the formulas from Theorem 2.2 are preserved if we replace $x$ by $-x$.

If $8 \mid x$ then $(x+p+5) / 8 \in \mathbb{Z}$ and $(x+p+5) / 8 \equiv(-x+p+5) / 8$ $(\bmod 2)$ so again the formulas from Theorem 2.2 make sense and they do not change if we replace $x$ by $-x$.

2.3. We will treat the two problems together. Note that $p \equiv 7,19(\bmod 24)$ means $p \equiv 7(\bmod 12)$. We have $p \equiv 1(\bmod 3)$ and the two cases, $p \equiv 3,19$ $(\bmod 24)$, correspond to $p \equiv 3,7(\bmod 8)$ respectively.

We have $2+\sqrt{3}=(1+\sqrt{3})^{2} / 2$. Let $\varepsilon=2+\sqrt{3}$ and $\varepsilon^{\prime}=1+\sqrt{3}$ and denote by $\bar{\varepsilon}, \bar{\varepsilon}^{\prime}$ their conjugates. We have $\left(\frac{3}{p}\right)=-1$ so $p$ is inert in $\mathbb{Q}(\sqrt{3})$. As in $\S 1$, we obtain $\varepsilon^{p+1} \equiv \varepsilon \bar{\varepsilon}=1(\bmod p)$ and $\varepsilon^{\prime p+1} \equiv \varepsilon^{\prime} \bar{\varepsilon}^{\prime}=-2(\bmod p)$. We reduce our problem to finding $\varepsilon^{\prime\left(p^{2}-1\right) / 8} \bmod p$.

If $p \equiv 7(\bmod 8)$ then $\varepsilon^{(p+1) / 8}=2^{-(p+1) / 8} \varepsilon^{\prime(p+1) / 4}$. But, as in 1.2 , we have

$$
\varepsilon^{\prime(p+1) / 4} \equiv \varepsilon^{\prime-\left(p^{2}-1\right) / 8}\left(\varepsilon^{\prime p+1}\right)^{(p+1) / 8} \equiv(-2)^{(p+1) / 8} \varepsilon^{-\left(p^{2}-1\right) / 2}(\bmod p) .
$$

It follows that

$$
\varepsilon^{(p+1) / 8} \equiv(-1)^{(p+1) / 8} \varepsilon^{\prime-\left(p^{2}-1\right) / 8}(\bmod p) .
$$

If $p \equiv 3(\bmod 8)$ then

$$
\varepsilon^{(p+5) / 8}=2^{-(p+5) / 8} \varepsilon^{(p+5) / 4}=2^{-(p+5) / 8}(1+\sqrt{3}) \varepsilon^{(p+1) / 4} .
$$

As in 1.2 , we also have

$$
\varepsilon^{\prime(p+1) / 4} \equiv \varepsilon^{\prime\left(p^{2}-1\right) / 8}\left(\varepsilon^{\prime p+1}\right)^{-(p-3) / 8} \equiv(-2)^{-(p-3) / 8} \varepsilon^{\prime\left(p^{2}-1\right) / 8}(\bmod p) .
$$

It follows that

$$
\varepsilon^{(p+5) / 8} \equiv(-1)^{(p-3) / 8} 2^{-(p+1) / 4}(1+\sqrt{3}) \varepsilon^{\prime\left(p^{2}-1\right) / 8}(\bmod p) .
$$

Theorem 2.2 can be stated as:

$$
\begin{aligned}
\varepsilon^{(p+5) / 8} & =V_{(p+5) / 8}+U_{(p+5) / 8} \sqrt{3} \\
& \equiv \begin{cases}(-1)^{(x+p+1) / 8} 6^{(p-3) / 4}\left(\frac{x}{3}\right)(3+\sqrt{3})(\bmod p) & \text { if } 8 \nmid x, \\
(-1)^{(x+p+5) / 8} 2^{(p-3) / 4}(1+\sqrt{3})(\bmod p) & \text { if } 8 \mid x .\end{cases}
\end{aligned}
$$


Since $\varepsilon^{(p+5) / 8} \equiv(-1)^{(p-3) / 8} 2^{-(p+1) / 4}(1+\sqrt{3}) \varepsilon^{\prime\left(p^{2}-1\right) / 8}(\bmod p)$ this is equivalent to

$$
\varepsilon^{\prime\left(p^{2}-1\right) / 8} \equiv \begin{cases}(-1)^{(x-4) / 8} 3^{(p-3) / 4}\left(\frac{x}{3}\right) \sqrt{3}(\bmod p) & \text { if } 8 \nmid x, \\ (-1)^{x / 8}(\bmod p) & \text { if } 8 \mid x .\end{cases}
$$

(Here we use the fact that $\left(\frac{2}{p}\right)=-1$ so $2^{(p+1) / 4} 6^{(p-3) / 4}=2^{(p-1) / 2} 3^{(p-3) / 4} \equiv$ $-3^{(p-3) / 4}(\bmod p)$ and $\left.2^{(p+1) / 4} 2^{(p-3) / 4}=2^{(p-1) / 2} \equiv-1(\bmod p).\right)$

From now on, the proof follows the pattern from $\S 1$. We write $p=u^{2}+2 v^{2}$ if $p \equiv 3(\bmod 8)$ and $p=u^{2}-2 v^{2}$ if $p \equiv 7(\bmod 8)$. Note that the relations $p=x^{2}+3 y^{2}=u^{2} \pm 2 v^{2}$ are similar to $2 p=x^{2}+5 y^{2}=u^{2} \pm 2 v^{2}$ from $\S 1$. Therefore we can repeat the definitions and results from $\S 1$ with $\mathbb{Q}(\sqrt{5}), 2 p$ and $x \pm y \sqrt{5} i$ replaced by $\mathbb{Q}(\sqrt{3}), p$ and $x \pm y \sqrt{3} i$. So we take $F=\mathbb{Q}(\sqrt{3})$ and $E=F(\zeta)=\mathbb{Q}(\sqrt{3}, \sqrt{2}, i)$. We define $L=E\left(\sqrt[8]{A_{1}}\right)$, where $A_{1} \in E$ is given by

$$
A_{1}= \begin{cases}p(x+y \sqrt{3} i)^{2}(u+v \sqrt{2} i)^{4} & \text { if } p \equiv 3(\bmod 8), \\ p(x+y \sqrt{3} i)^{6}(u+v \sqrt{2})^{4} & \text { if } p \equiv 7(\bmod 8) .\end{cases}
$$

$\operatorname{Again} \operatorname{Gal}(L / E)=\langle\sigma\rangle \cong \mathbb{Z}_{8}$ where $\sigma$ is given by $\sqrt[8]{A_{1}} \mapsto \zeta \sqrt[8]{A_{1}}$. For $k \in \mathbb{Z}_{8}^{\times}$ define $A_{k}$ and $\alpha_{k}$ similarly to the proof of Lemma 1.3. The analogue of Lemma 1.3 will hold so $\operatorname{Gal}(L / F) \cong \mathbb{Z}_{8}^{\times} \times \mathbb{Z}_{8}$. More precisely, $\operatorname{Gal}(L / F)$ is the internal direct product of its subgroups $H=\left\{\tau_{k} \mid k \in \mathbb{Z}_{8}^{\times}\right\}$and $\langle\sigma\rangle$.

Just as in $\S 1$ we define $\chi: \operatorname{Gal}(L / F) \rightarrow \mu_{8}$ by $\sigma^{k} \tau_{l} \mapsto \zeta^{k}$.

Define $\mathfrak{p}, \mathfrak{P}$ and $\infty_{ \pm}$as in $\S 1$. By a proof similar to that of Lemma 1.4 we have:

LEMMA 2.4.

$$
\begin{aligned}
\text { (i) } \chi\left(\left(\frac{\varepsilon^{\prime}, L / F}{\mathfrak{p}}\right)\right) & \equiv \varepsilon^{\prime-\left(p^{2}-1\right) / 8}(\bmod \mathfrak{P}) . \\
\text { (ii) } \chi\left(\left(\frac{\varepsilon^{\prime}, L / F}{\infty_{-}}\right)\right) & = \begin{cases}1 & \text { if } p \equiv 3(\bmod 8), \\
\operatorname{sgn}(u) & \text { if } p \equiv 7(\bmod 8) .\end{cases} \\
\text { (iii) } \chi\left(\left(\frac{\varepsilon^{\prime}, L / F}{\mathfrak{q}}\right)\right) & =1 \text { if } \mathfrak{q} \neq \mathfrak{p}, \infty_{-} \text {and } \mathfrak{q} \nmid 2 .
\end{aligned}
$$

(Note that for (ii) we use the fact that $\varepsilon_{\infty_{-}}^{\prime}=1-\sqrt{3}<0$, and for (iii) the fact that $\varepsilon_{\infty_{+}}^{\prime}=1+\sqrt{3}>0$ and $\varepsilon^{\prime} \mid 2$ so it is a unit at all nonarchimedian primes $\mathfrak{q}$ with $\mathfrak{q} \nmid 2$.)

So $\varepsilon^{\prime\left(p^{2}-1\right) / 8} \equiv \chi\left(\left(\frac{\varepsilon^{\prime}, L / F}{\mathfrak{q}}\right)\right)$ or $\operatorname{sgn}(u) \chi\left(\left(\frac{\varepsilon^{\prime}, L / F}{\mathfrak{q}}\right)\right)(\bmod \mathfrak{P})$, according as $p \equiv 3$ or $7(\bmod 8)$, where $\mathfrak{q}$ is the prime of $\mathbb{Q}(\sqrt{3})$ over 2 .

2.5. We now take another prime $p^{\prime} \equiv 7(\bmod 12)$ and let $x^{\prime}, y^{\prime}, u^{\prime}, v^{\prime}$ be the corresponding $x, y, u, v$. Assume that $p \equiv p^{\prime}(\bmod 16)$ and either $x \equiv x^{\prime}$ $(\bmod 16)$ or $x \equiv x^{\prime} \equiv 0(\bmod 8)$. 
Since $u^{2} \pm 2 v^{2}=x^{2}+3 y^{2}=p \equiv 3(\bmod 4)$ we see that $x$ is even and $y, u, v$ are odd, and similarly for $x^{\prime}, y^{\prime}, u^{\prime}, v^{\prime}$. By multiplying $y, u, v$ with \pm 1 we may assume that $y, u, v \equiv y^{\prime}, u^{\prime}, v^{\prime}(\bmod 4)$. This implies that $y+y^{\prime} \equiv$ $u+u^{\prime} \equiv v+v^{\prime} \equiv 2(\bmod 4)$.

Since $v, v^{\prime}$ are odd, we have $v^{2} \equiv v^{\prime 2} \equiv 1(\bmod 8)$. Together with $u^{2} \pm$ $2 v^{2}=p \equiv p^{\prime}=u^{\prime 2} \pm 2 v^{\prime 2}(\bmod 16)$, this implies $u^{2} \equiv u^{\prime 2}(\bmod 16)$. Since $16 \mid u^{\prime 2}-u^{2}$ and $u^{\prime}+u \equiv 2(\bmod 4)$, we get $8 \mid u^{\prime}-u$.

If $x \equiv x^{\prime}(\bmod 16)$ then $16 \mid x-x^{\prime}$ and $x+x^{\prime}$ is even so $32 \mid x^{2}-x^{\prime 2}$. The same happens if $x \equiv x^{\prime} \equiv 0(\bmod 8)$. This implies that $3\left(y^{\prime 2}-y^{2}\right)=$ $p^{\prime}-p+x^{2}-x^{\prime 2} \equiv p^{\prime}-p(\bmod 32)$. Since $16 \mid p^{\prime}-p$ we have $16 \mid y^{\prime 2}-y^{2}$ and $32 \mid y^{\prime 2}-y^{2}$ iff $32 \mid p^{\prime}-p$. But $y^{\prime}+y \equiv 2(\bmod 4)$ so $8 \mid y^{\prime}-y$ and $16 \mid y^{\prime}-y$ iff $32 \mid p^{\prime}-p$.

Also note that if $p \equiv 3(\bmod 8)$ then $x^{2}+3 y^{2}=p$ implies that $4 \mid x$, while if $p \equiv 7(\bmod 8)$ then $x \equiv 2(\bmod 4)$; and similarly for $x^{\prime}$. In particular, if $p \equiv p^{\prime} \equiv 7(\bmod 8)$ then $x \equiv x^{\prime}(\bmod 16)$ since we cannot have $x \equiv x^{\prime} \equiv 0$ $(\bmod 8)$.

We now prove the analogue of Lemma 1.8. Let $\mathfrak{Q}, \mathfrak{q}_{1}, \mathfrak{q}_{2}$ be the only primes of $E, \mathbb{Q}(\sqrt{3} i)$ and $\mathbb{Q}(\sqrt{2} i)$ or $\mathbb{Q}(\sqrt{2})$ lying over 2. Define $\widetilde{\mathfrak{Q}}, \widetilde{\mathfrak{q}}_{1}, \widetilde{\mathfrak{q}}_{2}$ and $\widetilde{2}$ as in $\S 1$.

Lemma 2.6. If $A_{1}^{\prime}$ is the $A_{1}$ corresponding to $p^{\prime}$, then $A_{1}^{\prime}=\left(\sqrt{3}^{s} t\right)^{8} A_{1}$ for some $t \in 1+2 \widetilde{\mathfrak{Q}}$, where $s=0$ if $x \equiv x^{\prime}(\bmod 16)$ and $s=1$ otherwise.

Proof. Note that $u+\sqrt{2} i$ (or $u+v \sqrt{2}$ ), $x+y \sqrt{3} i \mid p$, which is odd, so $u+\sqrt{2} i$ (or $u+v \sqrt{2}$ ), $x+y \sqrt{3} i, p$ are units in $\mathcal{O}_{\mathfrak{q}_{2}}, \mathcal{O}_{\mathfrak{q}_{1}}, \mathcal{O}_{2}$ respectively.

Suppose that $p \equiv 3(\bmod 8)$. Then $\mathfrak{q}_{2}$ is generated by $\sqrt{2} i$. Since $8 \mid u^{\prime}-u$ and $4 \mid v^{\prime}-v$, we have $\left(u^{\prime}+v^{\prime} \sqrt{2} i\right)-(u+v \sqrt{2} i)=\left(u^{\prime}-u\right)+\left(v^{\prime}-v\right) \sqrt{2} i \in 4 \mathfrak{q}_{2}$. Since also $u+v \sqrt{2} i \in \mathcal{O}_{\mathfrak{q}_{2}}^{\times}$we get

$$
\frac{u^{\prime}+v^{\prime} \sqrt{2} i}{u+v \sqrt{2} i}-1 \in 4 \widetilde{\mathfrak{q}}_{2}
$$

By Lemma 1.6(i) we have

$$
\frac{u^{\prime}+v^{\prime} \sqrt{2} i}{u+v \sqrt{2} i}=t_{1}^{2} \quad \text { for some } t_{1} \in 1+2 \widetilde{\mathfrak{q}}_{2} \subset 1+2 \widetilde{\mathfrak{Q}} .
$$

Similarly for $\frac{u^{\prime}+v^{\prime} \sqrt{2}}{u+v \sqrt{2}}$ when $p \equiv 7(\bmod 8)$.

We have $x \equiv x^{\prime}(\bmod 8)$ and $y \equiv y^{\prime}(\bmod 8)$. If $x+y \equiv x^{\prime}+y^{\prime}(\bmod 16)$ then we can write $x-x^{\prime}=8 a$ and $y-y^{\prime}=8 b$ with $a+b$ even. This implies that $\mathrm{N}_{\mathbb{Q}(\sqrt{3} i)_{\mathfrak{q}_{1} / \mathbb{Q}_{2}}}(a+b \sqrt{3} i)=a^{2}+3 b^{2}$ is even so $a+b \sqrt{3} i \in \mathfrak{q}_{1}$ so $\left(x^{\prime}+y^{\prime} \sqrt{3} i\right)-(x+y \sqrt{3} i)=8(a+b \sqrt{3} i) \in 8 \widetilde{\mathfrak{q}}_{1}$. Since also $x+y \sqrt{3} i \in \mathcal{O}_{\mathfrak{q}_{1}}^{\times}$ 
we have

$$
\frac{x^{\prime}+y^{\prime} \sqrt{3} i}{x+y \sqrt{3} i}-1 \in 8 \widetilde{\mathfrak{q}}_{1} .
$$

By Lemma 1.6(i) applied to $k=\mathbb{Q}(\sqrt{3} i)_{\mathfrak{q}_{1}}$ we get

$$
\frac{x^{\prime}+y^{\prime} \sqrt{3} i}{x+y \sqrt{3} i}=t_{2}^{4} \quad \text { for some } t_{2} \in 1+2 \widetilde{\mathfrak{q}}_{1} \subset 1+2 \widetilde{\mathfrak{Q}} .
$$

If $x+y \not \equiv x^{\prime}+y^{\prime}(\bmod 16)$ then $x^{\prime}+y^{\prime} \equiv x+y+8(\bmod 16)$. Note that $x+y$ is odd so $9 x+9 y \equiv x+y+8 \equiv x^{\prime}+y^{\prime}(\bmod 16)$. Since also $9 x \equiv x \equiv x^{\prime}$ $(\bmod 8)$ and $9 y \equiv y \equiv y^{\prime}(\bmod 8)$, by a similar reasoning to the one above, we get

$$
\frac{x^{\prime}+y^{\prime} \sqrt{3} i}{9 x+9 y \sqrt{3} i}=t_{2}^{4}, \quad \text { so } \quad \frac{x^{\prime}+y^{\prime} \sqrt{3} i}{x+y \sqrt{3} i}=9 t_{2}^{4}, \quad \text { for some } t_{2} \in 1+2 \widetilde{\mathfrak{Q}} .
$$

In conclusion,

$$
\frac{x^{\prime}+y^{\prime} \sqrt{3} i}{x+y \sqrt{3} i}=3^{2 s_{2}} t_{2}^{4} \quad \text { with } t_{2} \in 1+2 \widetilde{\mathfrak{Q}},
$$

where $s_{2}=0$ if $x+y \equiv x^{\prime}+y^{\prime}(\bmod 16)$ and $s_{2}=1$ if $x+y \equiv x^{\prime}+y^{\prime}+8$ $(\bmod 16)$.

If $p \equiv p^{\prime}(\bmod 32)$ then $p^{\prime}-p \in 32 \mathcal{O}_{2}=16 \widetilde{2}$, which, together with $p \in \mathcal{O}_{2}^{\times}$, implies that $p^{\prime} / p-1 \in 16 \widetilde{2}$. By Lemma 1.6(i) applied to $k=\mathbb{Q}_{2}$ we get $p^{\prime} / p=t_{3}^{8}$ for some $t_{3} \in 1+2 \widetilde{2} \subset 1+2 \widetilde{\mathfrak{Q}}$. If $p \not \equiv p^{\prime}(\bmod 32)$ then $p^{\prime} \equiv p+16(\bmod 16)$. But $p$ is odd so $81 p \equiv p+80 \equiv p+16 \equiv p^{\prime}(\bmod 32)$. As in the previous case, we get $p^{\prime} / 81 p=t_{3}^{8}$, so $p^{\prime} / p=81 t_{3}^{8}$ for some $t_{3} \in 1+2 \widetilde{\mathfrak{Q}}$. For short, $p^{\prime} / p=3^{4 s_{3}} t_{3}^{8}$ with $t_{3} \in 1+2 \widetilde{\mathfrak{Q}}$, where $s_{3}=0$ if $p \equiv p^{\prime}(\bmod 32)$ and $s_{3}=1$ if $p \equiv p^{\prime}+16(\bmod 32)$.

If $p \equiv 3(\bmod 8)$ then

$$
\begin{aligned}
\frac{A_{1}^{\prime}}{A_{1}} & =\frac{p^{\prime}}{p}\left(\frac{x^{\prime}+y^{\prime} \sqrt{3} i}{x+y \sqrt{3} i}\right)^{2}\left(\frac{u^{\prime}+v^{\prime} \sqrt{2} i}{u+v \sqrt{2} i}\right)^{4} \\
& =3^{4 s_{3}} t_{3}^{8}\left(3^{2 s_{2}} t_{2}^{4}\right)^{2}\left(t_{1}^{2}\right)^{4}=3^{4\left(s_{3}+s_{2}\right)}\left(t_{3} t_{2} t_{1}\right)^{8} .
\end{aligned}
$$

If $p \equiv 7(\bmod 8)$ then

$$
\begin{aligned}
\frac{A_{1}^{\prime}}{A_{1}} & =\frac{p^{\prime}}{p}\left(\frac{x^{\prime}+y^{\prime} \sqrt{3} i}{x+y \sqrt{3} i}\right)^{6}\left(\frac{u^{\prime}+v^{\prime} \sqrt{2}}{u+v \sqrt{2}}\right)^{4} \\
& =3^{4 s_{3}} t_{3}^{8}\left(3^{2 s_{2}} t_{2}^{4}\right)^{6}\left(t_{1}^{2}\right)^{4}=3^{4\left(s_{3}+3 s_{2}\right)}\left(t_{3} t_{2}^{3} t_{1}\right)^{8} .
\end{aligned}
$$

By 2.5 we have either $p \equiv p^{\prime}(\bmod 32)$ and $y \equiv y^{\prime}(\bmod 16)$, or $p \equiv p^{\prime}+16$ $(\bmod 32)$ and $y \equiv y^{\prime}+8(\bmod 16)$. We now consider separately the cases $x \equiv x^{\prime}(\bmod 16)$ and $x \equiv x^{\prime}+8(\bmod 16)$. 
If $x \equiv x^{\prime}(\bmod 16)$ then either $p \equiv p^{\prime}(\bmod 32)$ and $x+y \equiv x^{\prime}+y^{\prime}$ $(\bmod 16)$, or $p \equiv p^{\prime}+16(\bmod 32)$ and $x+y \equiv x^{\prime}+y^{\prime}+8(\bmod 16)$. This implies that either $s_{3}=s_{2}=0$ or $s_{3}=s_{2}=1$. If $p \equiv 3(\bmod 8)$ then

$$
\frac{A_{1}^{\prime}}{A_{1}}=3^{4\left(s_{3}+s_{2}\right)}\left(t_{3} t_{2} t_{1}\right)^{8}=\left\{\begin{array}{l}
\left(t_{3} t_{2} t_{1}\right)^{8} \text { or } \\
3^{8}\left(t_{3} t_{2} t_{1}\right)^{8}=\left(-3 t_{3} t_{2} t_{1}\right)^{8} .
\end{array}\right.
$$

If $p \equiv 7(\bmod 8)$ then

$$
\frac{A_{1}^{\prime}}{A_{1}}=3^{4\left(s_{3}+3 s_{2}\right)}\left(t_{3} t_{2}^{3} t_{1}\right)^{8}=\left\{\begin{array}{l}
\left(t_{3} t_{2}^{3} t_{1}\right)^{8} \text { or } \\
3^{16}\left(t_{3} t_{2}^{3} t_{1}\right)^{8}=\left(9 t_{3} t_{2}^{3} t_{1}\right)^{8} .
\end{array}\right.
$$

Thus $A_{1}^{\prime} / A_{1}=t^{8}$, where $t=t_{3} t_{2} t_{1},-3 t_{3} t_{2} t_{1}, t_{3} t_{2}^{3} t_{1}$ or $9 t_{3} t_{2}^{3} t_{1}$. But $t_{3}, t_{2}, t_{1} \in$ $1+2 \widetilde{\mathfrak{Q}}$ and also $-3,9 \in 1+4 \mathcal{O}_{2} \subset 1+2 \widetilde{\mathfrak{Q}}$. So in all four cases we have $t \in 1+2 \widetilde{\mathfrak{Q}}$.

If $x \equiv x^{\prime}+8(\bmod 16)$ then either $p \equiv p^{\prime}(\bmod 32)$ and $x+y \equiv x^{\prime}+y^{\prime}+8$ $(\bmod 16)$, or $p \equiv p^{\prime}+16(\bmod 32)$ and $x+y \equiv x^{\prime}+y^{\prime}(\bmod 16)$. This implies that either $s_{3}=0, s_{2}=1$ or $s_{3}=1, s_{2}=0$. Since $x \not \equiv x^{\prime}(\bmod 16)$ we must have $x \equiv x^{\prime} \equiv 0(\bmod 8)$ so $p=x^{2}+3 y^{2} \equiv 3(\bmod 8)$. We get

$$
\frac{A_{1}^{\prime}}{A_{1}}=3^{4\left(s_{3}+s_{2}\right)}\left(t_{3} t_{2} t_{1}\right)^{8}=3^{4}\left(t_{3} t_{2} t_{1}\right)^{8}=(\sqrt{3} t)^{8}, \quad \text { where } \quad t=t_{3} t_{2} t_{1} .
$$

But $t_{3}, t_{2}, t_{1}$ belong to $1+2 \widetilde{\mathfrak{Q}}$ and so does $t$.

Let $E^{\prime}, \chi^{\prime}$ be the $E, \chi$ corresponding to $p^{\prime}$.

LEMMA 2.7. We have

$$
\chi\left(\left(\frac{\varepsilon^{\prime}, L / F}{\mathfrak{q}}\right)\right)=(-1)^{\left(x^{\prime}-x\right) / 8} \chi^{\prime}\left(\left(\frac{\varepsilon^{\prime}, L^{\prime} / F}{\mathfrak{q}}\right)\right) .
$$

(Note that if $p \equiv p^{\prime} \equiv 7(\bmod 8)$ then by $2.5, x \equiv x^{\prime}(\bmod 16)$ so the factor $(-1)^{\left(x^{\prime}-x\right) / 8}$ can be dropped in the formula above.)

Proof. Let $\phi=\chi\left(\left(\frac{\varepsilon^{\prime}, L / F}{\mathfrak{q}}\right)\right)$. We have $\mathrm{N}_{\mathbb{Q}(\sqrt{3})_{\mathfrak{q}} / \mathbb{Q}_{2}}\left(\varepsilon^{\prime}\right)=-2$ so

$$
\left(\frac{\varepsilon^{\prime},-1}{\mathfrak{q}}\right)=\left(\frac{-2,-1}{2}\right)=-1, \quad\left(\frac{\varepsilon^{\prime}, 2}{\mathfrak{q}}\right)=\left(\frac{-2,2}{2}\right)=1 .
$$

Thus $\phi(i)=-i$ and $\phi(\sqrt{2})=\sqrt{2}$ and so $\phi(\zeta)=\bar{\zeta}=\zeta^{7}$.

Now the proof follows that of Lemma 1.9. We have $A_{1}^{\prime}=B^{8} A_{1}$, where $B=\sqrt{3}^{s} t$. If $\chi\left(\left(\frac{\varepsilon^{\prime}, L / F}{\mathfrak{q}}\right)\right)=\zeta^{k}$ and $\chi^{\prime}\left(\left(\frac{\varepsilon^{\prime}, L^{\prime} / F}{\mathfrak{q}}\right)\right)=\zeta^{k^{\prime}}$, then we have to prove that $\zeta^{k^{\prime}-k}=(-1)^{\left(x^{\prime}-x\right) / 8}$. By the same proof as for Lemma 1.9 we have

$$
\zeta^{k^{\prime}-k}=\frac{p^{\prime}}{p}(B \phi(B))^{-1} \quad \text { or } \quad \frac{p^{\prime}}{p} \cdot \frac{u^{\prime}+v^{\prime} \sqrt{2}}{u+v \sqrt{2}}(B \phi(B))^{-1},
$$


according as $p \equiv 3$ or $7(\bmod 8)$. Now $p \equiv p^{\prime}(\bmod 16)$, so $p^{\prime} / p \in 1+16 \mathcal{O}_{2} \subset$ $1+2 \widetilde{\mathfrak{Q}}$, and if $p \equiv 7(\bmod 8)$, then

$$
\frac{u^{\prime}+v^{\prime} \sqrt{2}}{u+v \sqrt{2}} \in 1+4 \widetilde{\mathfrak{q}}_{2} \subset 1+2 \widetilde{\mathfrak{Q}} .
$$

On the other hand, $B=\sqrt{3}^{s} t$ so $B \phi(B)=3^{s} t \phi(t)$. By Lemma 2.6, $t$ belongs to $1+2 \widetilde{\mathfrak{Q}}$ and so does its conjugate $\phi(t)$. In the case $x \equiv x^{\prime}(\bmod 16)$, when $(-1)^{\left(x^{\prime}-x\right) / 8}=1$, we have $s=0$ so $(B \phi(B))^{-1}=(t \phi(t))^{-1} \in 1+2 \widetilde{\mathfrak{Q}}$. This implies that $\zeta^{k^{\prime}-k} \in 1+2 \widetilde{\mathfrak{Q}}$ and so $\zeta^{k^{\prime}-k}=1$. In the case $x \equiv x^{\prime}+8$ $(\bmod 16)$, when $(-1)^{\left(x^{\prime}-x\right) / 8}=-1$, we have $s=1$ so $3(B \phi(B))^{-1}=$ $(t \phi(t))^{-1} \in 1+2 \widetilde{\mathfrak{Q}}$. It follows that $3 \zeta^{k^{\prime}-k} \in 1+2 \widetilde{\mathfrak{Q}}$. Together with $4 \zeta^{k^{\prime}-k} \in$ $4 \mathcal{O}_{\mathfrak{Q}} \subset 2 \widetilde{\mathfrak{Q}}$, this implies by subtraction that $-\zeta^{k^{\prime}-k} \in 1+2 \widetilde{\mathfrak{Q}}$ and so $-\zeta^{k^{\prime}-k}=1$.

LEMMA 2.8.

(i) If $p \equiv 3(\bmod 8)$ then

$$
\left(\frac{x}{p}\right)=\left(\frac{x}{3}\right), \quad\left(\frac{y}{p}\right)=\left(\frac{y, 3}{2}\right) .
$$

(ii) If $p \equiv 7(\bmod 8)$ then

$$
\begin{aligned}
\left(\frac{x}{p}\right) & =\left(\frac{x, 5}{2}\right)\left(\frac{x}{3}\right), & \left(\frac{y}{p}\right) & =\left(\frac{y, 7}{2}\right), \\
\left(\frac{u}{p}\right) & =\left(\frac{u, 2}{2}\right) \operatorname{sgn}(u), & \left(\frac{v}{p}\right) & =\left(\frac{v, 7}{2}\right) .
\end{aligned}
$$

Proof. As in the proof of Lemma 1.10, the relation $x^{2}+3 y^{2}=p$ implies $\left(\frac{x, 3 p}{q}\right)=1$ for $q \neq 2,3, p$ and $\left(\frac{y, p}{q}\right)=1$ for $q \neq 2, p$. Also $\left(\frac{x, 3 p}{\infty}\right)=\left(\frac{y, p}{\infty}\right)=1$. Therefore$$
\left(\frac{x}{p}\right)=\left(\frac{x, 3 p}{p}\right)=\left(\frac{x, 3 p}{2}\right)\left(\frac{x, 3 p}{3}\right)=\left(\frac{x, 3 p}{2}\right)\left(\frac{x}{3}\right),\left(\frac{y}{p}\right)=\left(\frac{y, p}{p}\right)=\left(\frac{y, p}{2}\right) .
$$

If $p \equiv 3(\bmod 8)$ then $\left(\frac{x, 3 p}{2}\right)=1$ and $\left(\frac{y, p}{2}\right)=\left(\frac{y, 3}{2}\right)$. If $p \equiv 7(\bmod 8)$ then $\left(\frac{x, 3 p}{2}\right)=\left(\frac{x, 5}{2}\right)$ and $\left(\frac{y, p}{2}\right)=\left(\frac{y, 7}{2}\right)$. This yields the formulas for $\left(\frac{x}{p}\right)$ and $\left(\frac{y}{p}\right)$ from (i) and (ii).

If $p \equiv 7(\bmod 8)$ then $u^{2}-2 v^{2}=p$ implies $\left(\frac{u,-2 p}{q}\right)=1$ and $\left(\frac{v, p}{q}\right)=1$ for $q \neq 2, p$. Also $\left(\frac{v, p}{\infty}\right)=1$. Hence

$$
\begin{gathered}
\left(\frac{u}{p}\right)=\left(\frac{u,-2 p}{p}\right)=\left(\frac{u,-2 p}{2}\right)\left(\frac{u,-2 p}{\infty}\right)=\left(\frac{u, 2}{2}\right) \operatorname{sgn}(u), \\
\left(\frac{v}{p}\right)=\left(\frac{v, p}{2}\right)=\left(\frac{v, 7}{2}\right) .
\end{gathered}
$$


Lemma 2.9. Let $s, t \in \mathcal{O}_{p}, \alpha, \beta \in\{ \pm 1\}$.

(i) If $p \equiv 7(\bmod 8)$ and $s+t \sqrt{3} \equiv \frac{\alpha+\beta i}{\sqrt{2}}(\bmod \widetilde{\mathfrak{P}})$ then

$$
s \equiv\left(\frac{s}{p}\right) 2^{(p-3) / 4}(\bmod p) \quad \text { and } \quad t \equiv-\left(\frac{t}{p}\right) 6^{(p-3) / 4}(\bmod p) .
$$

Also

$$
\left(\frac{s}{p}\right)=\left(\frac{u v}{p}\right) \alpha \quad \text { and } \quad\left(\frac{t}{p}\right)=-\left(\frac{x y u v}{p}\right) \beta .
$$

(ii) If $p \equiv 3(\bmod 8)$ and $s+t \sqrt{3} \equiv \beta i(\bmod \widetilde{\mathfrak{P}})$ then $s \equiv 0(\bmod p)$ and $t \equiv\left(\frac{t}{p}\right) 3^{(p-3) / 4}(\bmod p)$. Also $\left(\frac{t}{p}\right)=-\left(\frac{x y}{p}\right) \beta$.

Proof. (i) We have $x-y \sqrt{3} i, u-v \sqrt{2} \in \widetilde{\mathfrak{P}}$ so $x \equiv y \sqrt{3} i(\bmod \widetilde{\mathfrak{P}})$ and $u \equiv v \sqrt{2}(\bmod \widetilde{\mathfrak{P}})$. Since also $p \nmid x y u v$, we have

$$
\frac{1}{\sqrt{2}} \equiv \frac{v}{u}(\bmod \widetilde{\mathfrak{P}}) \quad \text { and } \quad \frac{i}{\sqrt{2}} \equiv-\frac{y v}{x u} \sqrt{3}(\bmod \widetilde{\mathfrak{P}})
$$

Therefore

$$
s+t \sqrt{3} \equiv \frac{\alpha+\beta i}{\sqrt{2}} \equiv \alpha \frac{v}{u}-\beta \frac{y v}{x u} \sqrt{3}(\bmod \widetilde{\mathfrak{P}}) .
$$

Since both sides belong to $F_{\mathfrak{p}}$, the congruence will also hold modulo $\widetilde{\mathfrak{p}}$ so

$$
s \equiv \alpha \frac{v}{u}(\bmod p), \quad t \equiv-\beta \frac{y v}{x u}(\bmod p) .
$$

By Remark 1.11 we get

$$
\left(\frac{s}{p}\right)=\left(\frac{u v \alpha}{p}\right)=\left(\frac{u v}{p}\right) \alpha, \quad\left(\frac{t}{p}\right)=\left(\frac{-x y u v \beta}{p}\right)=-\left(\frac{x y u v}{p}\right) \beta .
$$

We have

$$
s \equiv \alpha \frac{v}{u} \equiv \frac{\alpha}{\sqrt{2}}(\bmod \tilde{\mathfrak{P}}), \quad t \sqrt{3} \equiv-\beta \frac{y v}{x u} \sqrt{3} \equiv \frac{\beta i}{\sqrt{2}}(\bmod \widetilde{\mathfrak{P}})
$$

so $s^{2} \equiv \frac{1}{2}(\bmod \widetilde{\mathfrak{P}})$ and $3 t^{2} \equiv-\frac{1}{2}(\bmod \widetilde{\mathfrak{P}})$. Since both sides belong to $\mathbb{Q}_{p}$, these congruences also hold modulo $p$. Consequently, $s^{2} \equiv \frac{1}{2}(\bmod p)$ and $t^{2} \equiv-\frac{1}{6}(\bmod p)$. It follows that $\left(\frac{2}{p}\right)=\left(\frac{-6}{p}\right)=1$ so $2^{(p-1) / 2} \equiv(-6)^{(p-1) / 2} \equiv$ $1(\bmod p)$. Therefore $s^{2} \equiv \frac{1}{2} \equiv\left(2^{(p-3) / 4}\right)^{2}(\bmod p)$ and so $s \equiv \pm 2^{(p-3) / 4}$ $(\bmod p)$. By Remark 1.11 we have

$$
s \equiv\left(\frac{2^{(p-3) / 4} s}{p}\right) 2^{(p-3) / 4}=\left(\frac{s}{p}\right) 2^{(p-3) / 4}(\bmod p) .
$$

Similarly

$$
t \equiv\left(\frac{t}{p}\right)(-6)^{(p-3) / 4}=-\left(\frac{t}{p}\right) 6^{(p-3) / 4}(\bmod p) .
$$

(Note that $p \equiv 7(\bmod 8)$ so $(p-3) / 4$ is odd.) 
(ii) The congruence $x \equiv y \sqrt{3} i(\bmod \widetilde{\mathfrak{P}})$ implies $i \equiv-\frac{y}{x} \sqrt{3}(\bmod \widetilde{\mathfrak{P}})$ so $s+t \sqrt{3} \equiv-\beta \frac{y}{x} \sqrt{3}(\bmod \widetilde{\mathfrak{P}})$. It follows that $s \equiv 0(\bmod p)$ and $t \equiv-\beta \frac{y}{x}$ $(\bmod p)$. By Remark 1.11,

$$
\left(\frac{t}{p}\right)=\left(\frac{-x y \beta}{p}\right)=-\left(\frac{x y}{p}\right) \beta .
$$

We have $t \sqrt{3} \equiv-\beta \frac{y}{x} \sqrt{3} \equiv \beta i(\bmod \widetilde{\mathfrak{P}})$ so $3 t^{2} \equiv-1(\bmod \widetilde{\mathfrak{P}})$. This implies $t^{2} \equiv-\frac{1}{3}(\bmod p)$. We have $\left(\frac{-3}{p}\right)=1$ so $(-3)^{(p-1) / 2} \equiv 1(\bmod p)$. Thus $t^{2} \equiv-\frac{1}{3} \equiv\left((-3)^{(p-3) / 4}\right)^{2}(\bmod p)$ so $t \equiv \pm(-3)^{(p-3) / 4}(\bmod p)$. By Remark 1.11 we have

$$
t \equiv\left(\frac{(-3)^{(p-3) / 4} t}{p}\right)(-3)^{(p-3) / 4}=\left(\frac{t}{p}\right) 3^{(p-3) / 4}(\bmod p) .
$$

(Note that $p \equiv 3(\bmod 8)$ so $(p-3) / 4$ is even.)

2.10. By 2.3 we have $\varepsilon^{\prime p+1} \equiv-2(\bmod p)$ so

$$
\varepsilon^{\prime\left(p^{2}-1\right) / 2} \equiv(-2)^{(p-1) / 2}=\left(\frac{-2}{p}\right)(\bmod p)
$$

and this congruence also holds modulo $\widetilde{\mathfrak{P}}$. If $p \equiv 3(\bmod 8)$ then $\varepsilon^{\prime\left(p^{2}-1\right) / 2} \equiv$ $1(\bmod \widetilde{\mathfrak{P}})$ so $\varepsilon^{\prime\left(p^{2}-1\right) / 8} \equiv \eta(\bmod \widetilde{\mathfrak{P}})$ for some $\eta \in \mu_{4}$. If $p \equiv 7(\bmod 8)$ then $\varepsilon^{\prime\left(p^{2}-1\right) / 2} \equiv-1(\bmod \widetilde{\mathfrak{P}})$ so $\varepsilon^{\prime\left(p^{2}-1\right) / 8} \equiv \eta(\bmod \widetilde{\mathfrak{P}})$ for some primitive $\eta \in \mu_{8}$.

If $p \equiv 3(\bmod 8)$ then by Lemma 2.4 we have

$$
\varepsilon^{\prime\left(p^{2}-1\right) / 2} \equiv \chi\left(\left(\frac{\varepsilon^{\prime}, L / F}{\mathfrak{q}}\right)\right)(\bmod \widetilde{\mathfrak{P}})
$$

so $\eta=\chi\left(\left(\frac{\varepsilon^{\prime}, L / F}{\mathfrak{q}}\right)\right)$. (If $\eta, \eta^{\prime} \in \mu_{8}$ and $\eta \equiv \eta^{\prime}(\bmod \widetilde{\mathfrak{P}})$ then $\eta=\eta^{\prime}$ since otherwise $\eta-\eta^{\prime} \mid 2$ so $\eta-\eta^{\prime} \notin \widetilde{\mathfrak{P}}$.) Similarly if $\mathfrak{p} \equiv 7(\bmod 8)$ then $\eta=$ $\operatorname{sgn}(u) \chi\left(\left(\frac{\varepsilon^{\prime}, L / F}{\mathfrak{q}}\right)\right)$. It follows that $\chi\left(\left(\frac{\varepsilon^{\prime}, L / F}{\mathfrak{q}}\right)\right)$ is primitive in $\mu_{8}$ if $p \equiv 3$ $(\bmod 8)$ and it belongs to $\mu_{4}$ if $p \equiv 7(\bmod 8)$.

Lemma 2.11. If $p \equiv 7(\bmod 24)$ then

$$
\begin{aligned}
U_{(p+1) / 8} & \equiv-\left(\frac{U_{(p+1) / 8}}{p}\right) 6^{(p-3) / 4}(\bmod p), \\
V_{(p+1) / 8} & \equiv\left(\frac{V_{(p+1) / 8}}{p}\right) 2^{(p-3) / 4}(\bmod p) .
\end{aligned}
$$

Also

$$
\left(\frac{U_{(p+1) / 8}}{p}\right)=A\left(\frac{x}{3}\right), \quad\left(\frac{V_{(p+1) / 8}}{p}\right)=B,
$$

where $A$ and $B$ are two functions of $p \bmod 16$ and $x \bmod 16, A$ is odd and $B$ is even in the variable $x$. 
Proof. We have

$$
\varepsilon^{\prime\left(p^{2}-1\right) / 8} \equiv \operatorname{sgn}(u) \chi\left(\left(\frac{\varepsilon^{\prime}, L / F}{\mathfrak{q}}\right)\right)(\bmod \widetilde{\mathfrak{P}})
$$

and, by $2.3, \varepsilon^{(p+1) / 8} \equiv(-1)^{(p+1) / 8} \varepsilon^{\prime-\left(p^{2}-1\right) / 8}(\bmod p)$. Thus

$$
\varepsilon^{(p+1) / 8} \equiv \operatorname{sgn}(u)(-1)^{(p+1) / 8} \chi\left(\left(\frac{\varepsilon^{\prime}, L / F}{\mathfrak{q}}\right)\right)^{-1}(\bmod \widetilde{\mathfrak{P}}) .
$$

By 2.10, $(-1)^{(p+1) / 8} \chi\left(\left(\frac{\varepsilon^{\prime}, L / F}{\mathfrak{q}}\right)\right)^{-1}$ is primitive in $\mu_{8}$ so it can be written as $(\alpha+\beta i) / \sqrt{2}$ for some $\alpha, \beta \in\{ \pm 1\}$. Hence

$$
V_{(p+1) / 8}+U_{(p+1) / 8} \sqrt{3}=\varepsilon^{(p+1) / 8} \equiv \frac{\alpha \operatorname{sgn}(u)+\beta \operatorname{sgn}(u) i}{\sqrt{2}}(\bmod \widetilde{\mathfrak{P}}) .
$$

By Lemma 2.9(i) we have

$$
\begin{aligned}
V_{(p+1) / 8} & \equiv\left(\frac{V_{(p+1) / 8}}{p}\right) 2^{(p-3) / 4}(\bmod p), \\
U_{(p+1) / 8} & \equiv-\left(\frac{U_{(p+1) / 8}}{p}\right) 6^{(p-3) / 4}(\bmod p) .
\end{aligned}
$$

Also

$$
\begin{aligned}
\left(\frac{V_{(p+1) / 8}}{p}\right) & =\left(\frac{u v}{p}\right) \alpha \operatorname{sgn}(u)=B, \\
\left(\frac{U_{(p+1) / 8}}{p}\right) & =-\left(\frac{x y u v}{p}\right) \beta \operatorname{sgn}(u)=A\left(\frac{x}{3}\right),
\end{aligned}
$$

where

$$
B=\left(\frac{u, 2}{2}\right)\left(\frac{v, 7}{2}\right) \alpha, \quad A=-\left(\frac{x, 5}{2}\right)\left(\frac{y, 7}{2}\right)\left(\frac{u, 2}{2}\right)\left(\frac{v, 7}{2}\right) \beta .
$$

(See Lemma 2.8(ii).)

We now prove that $A, B$ depend only on $p \bmod 16$ and $x \bmod 16$. Let $p^{\prime} \equiv 7(\bmod 24)$ be another prime such that $p^{\prime} \equiv p(\bmod 16)$ and $x^{\prime} \equiv x$ $(\bmod 16)$. We keep the reductions of 2.5. Let $\alpha^{\prime}, \beta^{\prime}, A^{\prime}, B^{\prime}$ be the $\alpha, \beta, A, B$ corresponding to $p^{\prime}$. In order to prove that $A^{\prime}=A$ we show that the factors of $A^{\prime}$ are equal to the similar factors of $A$, and the same for $B^{\prime}=B$. By Lemma 2.7 we have $\chi^{\prime}\left(\left(\frac{\varepsilon^{\prime}, L^{\prime} / F}{\mathfrak{q}}\right)\right)=\chi\left(\left(\frac{\varepsilon^{\prime}, L / F}{\mathfrak{q}}\right)\right)$. Since $p \equiv p^{\prime}(\bmod 16)$ we also have $(-1)^{\left(p^{\prime}+1\right) / 8}=(-1)^{(p+1) / 8}$ and so

$$
(-1)^{\left(p^{\prime}+1\right) / 8} \chi^{\prime}\left(\left(\frac{\varepsilon^{\prime}, L^{\prime} / F}{\mathfrak{q}}\right)\right)=(-1)^{(p+1) / 8} \chi\left(\left(\frac{\varepsilon^{\prime}, L / F}{\mathfrak{q}}\right)\right),
$$


which implies that $\alpha^{\prime}=\alpha$ and $\beta^{\prime}=\beta$. We still have to prove that

$$
\begin{array}{ll}
\left(\frac{x^{\prime}, 5}{2}\right)=\left(\frac{x, 5}{2}\right), & \left(\frac{y^{\prime}, 7}{2}\right)=\left(\frac{y, 7}{2}\right), \\
\left(\frac{u^{\prime}, 2}{2}\right)=\left(\frac{u, 2}{2}\right), & \left(\frac{v^{\prime}, 7}{2}\right)=\left(\frac{v, 7}{2}\right),
\end{array}
$$

i.e. that

$$
\left(\frac{x x^{\prime}, 5}{2}\right)=\left(\frac{y y^{\prime}, 7}{2}\right)=\left(\frac{u u^{\prime}, 2}{2}\right)=\left(\frac{v v^{\prime}, 7}{2}\right)=1 .
$$

But this follows from $x x^{\prime}, y y^{\prime}, u u^{\prime} \in \mathbb{Q}_{2}^{\times 2}$ and $v v^{\prime} \in \mathbb{Q}_{2}^{\times 2} \cup 5 \mathbb{Q}_{2}^{\times 2}$. (By $2.5, x / 2, x^{\prime} / 2, y, y^{\prime}, u, u^{\prime}, v, v^{\prime}$ are all odd, and $x / 2 \equiv x^{\prime} / 2(\bmod 8), y \equiv y^{\prime}$ $(\bmod 8), u \equiv u^{\prime}(\bmod 8)$ and $\left.v \equiv v^{\prime}(\bmod 4).\right)$

Finally, note that if $x^{2}+3 y^{2}=p$ then also $(-x)^{2}+3 y^{2}=p$. Since $\left(\frac{U_{(p+1) / 8}}{p}\right)$ and $\left(\frac{V_{(p+1) / 8}}{p}\right)$ are independent of how we write $p$ as $x^{2}+3 y^{2}$ we must have $A(x, p)\left(\frac{x}{3}\right)=A(-x, p)\left(\frac{-x}{3}\right)$ and $B(x, p)=B(-x, p)$. So $A$ is odd and $B$ is even in the variable $x$.

Proof of Conjecture 2.1. With the notation of 2.3, we have to prove that $A=(-1)^{\left((x+4)^{2}-4\right) / 32}$ and $B=(-1)^{\left(x^{2}-4\right) / 32}$. It is easy to verify that the mappings $x \mapsto(-1)^{\left((x+4)^{2}-4\right) / 32}$ and $x \mapsto(-1)^{\left(x^{2}-4\right) / 32}$, defined on integers $x \equiv 2(\bmod 4)$, depend only on $x \bmod 16$, and they are odd and even, respectively. In view of Lemma 2.10, the two equalities need to be verified for a set of primes $p \equiv 7(\bmod 24)$ such that $p$ covers all possible remainders modulo 16 , namely 7,15 and $\pm x$ all possible remainders modulo 16 , namely \pm 2 and \pm 6 . But the primes $7,31,103,127$ cover all four possibilities. (We have $7=2^{2}+3 \cdot 1^{2}, 31=2^{2}+3 \cdot 3^{2}, 103=10^{2}+3 \cdot 1^{2}$ and $127=10^{2}+3 \cdot 3^{2}$.) It is easy to see that Sun's conjecture is true at these primes.

Proof of Theorem 2.2. Suppose that $p \equiv 19(\bmod p)$ and let $s+t \sqrt{3}=$ $\varepsilon^{\prime\left(p^{2}-1\right) / 8}$. By 2.3, Theorem 2.2 is equivalent to

$$
s+t \sqrt{3} \equiv \begin{cases}(-1)^{(x-4) / 8} 3^{(p-3) / 4}\left(\frac{x}{3}\right) \sqrt{3}(\bmod p) & \text { if } 8 \nmid x, \\ (-1)^{x / 8}(\bmod p) & \text { if } 8 \mid x .\end{cases}
$$

We have

$$
\varepsilon^{\prime\left(p^{2}-1\right) / 8} \equiv \chi\left(\left(\frac{\varepsilon^{\prime}, L / F}{\mathfrak{q}}\right)\right)(\bmod \widetilde{\mathfrak{P}}) .
$$

By 2.10, $\chi\left(\left(\frac{\varepsilon^{\prime}, L / F}{\mathfrak{q}}\right)\right) \in \mu_{4}$ so it equals $\alpha= \pm 1$ or $\beta i$ with $\beta= \pm 1$. In the first case $s+t \sqrt{3} \equiv \alpha(\bmod \widetilde{\mathfrak{P}})$ implies $s+t \sqrt{3} \equiv \alpha(\bmod p)$, as both sides belong to $F$. In the second case $s+t \sqrt{3} \equiv \alpha(\bmod \widetilde{\mathfrak{P}})$ implies by Lemmas 2.9 (ii) and $2.8(\mathrm{i})$ that $s \equiv 0(\bmod p)$ and 
SO

$$
t \equiv-\left(\frac{x y}{p}\right) 3^{(p-3) / 4} \beta=-\left(\frac{y, 3}{2}\right) \beta \cdot 3^{(p-3) / 4}\left(\frac{x}{3}\right)
$$

$$
s+t \sqrt{3} \equiv-\left(\frac{y, 3}{2}\right) \beta \cdot 3^{(p-3) / 4}\left(\frac{x}{3}\right) \sqrt{3}(\bmod p) .
$$

It follows that Theorem 2.2 is equivalent to

$$
\chi\left(\left(\frac{\varepsilon^{\prime}, L / F}{\mathfrak{q}}\right)\right)=\eta= \begin{cases}-(-1)^{(x-4) / 8}\left(\frac{y, 3}{2}\right) i & \text { if } 8 \nmid x, \\ (-1)^{x / 8} & \text { if } 8 \mid x .\end{cases}
$$

Let now $p^{\prime} \equiv 19(\bmod 24)$ be another prime. We use the notations from 2.5 and Lemma 2.7 .

Lemma 2.12. If $p \equiv p^{\prime}(\bmod 16)$ and $x \equiv x^{\prime}(\bmod 8)$ then the conclusion of Theorem 2.2 holds for $p$ iff it holds for $p^{\prime}$.

Proof. By 2.5 we have $4 \mid x, x^{\prime}$ so $x \equiv x^{\prime} \equiv 0$ or $4(\bmod 8)$. We consider the two cases.

If $x \equiv x^{\prime} \equiv 4(\bmod 8)$ then $x / 4, x^{\prime} / 4$ are odd integers. By multiplying $x$ with \pm 1 , we may assume that $x / 4 \equiv x^{\prime} / 4(\bmod 4)$ so $x \equiv x^{\prime}(\bmod 16)$. We also have $p \equiv p^{\prime}(\bmod 16)$ so we may apply the reductions of 2.5. By Lemma 2.7 we have $\chi\left(\left(\frac{\varepsilon^{\prime}, L / F}{\mathfrak{q}}\right)\right)=\chi^{\prime}\left(\left(\frac{\varepsilon^{\prime}, L^{\prime} / F}{\mathfrak{q}}\right)\right)$. Now Theorem 2.2 for $p$ and $p^{\prime}$ is equivalent to

$$
\begin{aligned}
\chi\left(\left(\frac{\varepsilon^{\prime}, L / F}{\mathfrak{q}}\right)\right) & =-(-1)^{(x-4) / 8}\left(\frac{y, 2}{p}\right) i, \\
\chi^{\prime}\left(\left(\frac{\varepsilon^{\prime}, L^{\prime} / F}{\mathfrak{q}}\right)\right) & =-(-1)^{\left(x^{\prime}-4\right) / 8}\left(\frac{y^{\prime}, 2}{p}\right) i,
\end{aligned}
$$

so in order to prove that the two statements are equivalent it is enough to prove that

$$
-(-1)^{(x-4) / 8}\left(\frac{y, 2}{p}\right) i=-(-1)^{\left(x^{\prime}-4\right) / 8}\left(\frac{y^{\prime}, 2}{p}\right) i .
$$

But $x \equiv x^{\prime}(\bmod 16)$ so $(-1)^{(x-4) / 8}=(-1)^{\left(x^{\prime}-4\right) / 8}$, so we still need $\left(\frac{y, 3}{2}\right)=$ $\left(\frac{y^{\prime}, 3}{2}\right)$. This follows from the fact that $y, y^{\prime}$ are odd and $y \equiv y^{\prime}(\bmod 8)$ so they are in the same square class in $\mathbb{Q}_{2}^{\times}$.

If $x \equiv x^{\prime} \equiv 0(\bmod 8)$ then again we can apply the reductions of 2.5 . Theorem 2.2 for $p$ and $p^{\prime}$ is equivalent to

$$
\chi\left(\left(\frac{\varepsilon^{\prime}, L / F}{\mathfrak{q}}\right)\right)=(-1)^{x / 8}, \quad \chi^{\prime}\left(\left(\frac{\varepsilon^{\prime}, L^{\prime} / F}{\mathfrak{q}}\right)\right)=(-1)^{x^{\prime} / 8} .
$$

The two statements are equivalent because by Lemma 2.7 we have

$$
\chi\left(\left(\frac{\varepsilon^{\prime}, L / F}{\mathfrak{q}}\right)\right)=(-1)^{\left(x^{\prime}-x\right) / 8} \chi^{\prime}\left(\left(\frac{\varepsilon^{\prime}, L^{\prime} / F}{\mathfrak{q}}\right)\right) .
$$


So it is enough to check Theorem 2.2 for a set of primes $p \equiv 19(\bmod 24)$ such that $p$ covers all the possible remainders modulo 16 , namely 3 and 11 , and $x$ covers all the possible remainders modulo 8 , namely 0 and 4 . The primes 19, 43, 67 and 139 cover all four possibilities. (We have $19=4^{2}+3 \cdot 1^{2}$, $43=4^{2}+3 \cdot 3^{2}, 67=8^{2}+3 \cdot 1^{2}$ and $139=8^{2}+3 \cdot 5^{2}$.)

3. Related problems. Throughout this section $d>1$ is a square-free integer and $\varepsilon$ is an integer of $\mathbb{Q}(\sqrt{d})$. For the time being we assume that $d>2$. If $p \equiv 3(\bmod 4)$ is a prime with $\left(\frac{d}{p}\right)=-1$ then $\left(\frac{-d}{p}\right)=1$ so $p$ can be written as $p=f(x, y)$ with $x, y \in \mathbb{Z}$, where $f(x, y)=a x^{2}+b x y+c y^{2}$ is a quadratic form with the discriminant $b^{2}-4 a c=-d$ or $-4 d$, according as $-d \equiv 1(\bmod 4)$ or $-d \equiv 2,3(\bmod 4)$. For any prime $q$ (including $q=\infty)$ we denote by $f_{q}$ the localized of $f$ at $q$.

We want to determine $\varepsilon^{(p+1) / 4} \bmod p$ in terms of $x$ and $y$. We could also determine the value modulo $p$ for $\varepsilon^{(p+1) / 8}$ if $p \equiv 7(\bmod 8)$ and for $\varepsilon^{(p+5) / 8}$ if $p \equiv 3(\bmod 8)$ assuming that $\varepsilon=\varepsilon_{d}$ is the fundamental unit of $\mathbb{Q}(\sqrt{d})$ and the norm of $\varepsilon$ is 1 . In this case, as in $\S 2$, we can write $\varepsilon=\varepsilon^{\prime 2} / m$ for some integer $\varepsilon^{\prime}$ in $\mathbb{Q}(\sqrt{d})$, and $m \in \mathbb{Z}^{\times}$. We can take for example $\varepsilon^{\prime}=1+\varepsilon$, and since $\varepsilon \bar{\varepsilon}=\mathrm{N} \varepsilon=1$, we have $\varepsilon=\varepsilon^{\prime} / \bar{\varepsilon}^{\prime}=\varepsilon^{\prime 2} / m$, where $m=\varepsilon^{\prime} \bar{\varepsilon}^{\prime}=\mathrm{N} \varepsilon^{\prime}$. Then if $p \equiv 7(\bmod 8)$ we have $\varepsilon^{(p+1) / 8}=m^{-(p+1) / 8} \varepsilon^{\prime(p+1) / 4}$, while if $p \equiv 3$ $(\bmod 8)$ then $\varepsilon^{(p+5) / 8}=m^{-(p+5) / 8} \varepsilon^{\prime} \varepsilon^{(p+1) / 4}$, so in both cases we have to determine $\varepsilon^{\prime(p+1) / 4} \bmod p$.

As in 1.2 or 2.3 , we reduce our problem to finding $\varepsilon^{\left(p^{2}-1\right) / 8} \bmod p$. Namely, we have $\varepsilon^{p+1} \equiv N(\bmod p)$ and so

$$
\varepsilon^{(p+1) / 4} \equiv \begin{cases}N^{-(p-3) / 8} \varepsilon^{\left(p^{2}-1\right) / 8}(\bmod p) & \text { if } p \equiv 3(\bmod p), \\ N^{(p+1) / 8} \varepsilon^{-\left(p^{2}-1\right) / 8}(\bmod p) & \text { if } p \equiv 7(\bmod p),\end{cases}
$$

where $N=\mathrm{N} \varepsilon=\varepsilon \bar{\varepsilon}$.

We consider the two cases $p \equiv 3$ or $7(\bmod 8)$ separately. Note that besides the condition that the discriminant $b^{2}-4 a c$ is $-d$ or $-4 d, f$ should also represent numbers $\equiv 3$ or $7(\bmod 8)$. This is equivalent to the fact that $f_{2}$ represents 3 or -1 , respectively. We claim that the rational quadratic form $F(x, y, u, v)=f(x, y)-\left(u^{2} \pm 2 v^{2}\right)$, where the sign is + if $p \equiv 3(\bmod 8)$ and - if $p \equiv 7(\bmod 8)$, is isotropic. By the Hasse-Minkowski theorem we have to prove this statement locally. Since $f$ is positive definite $F$ will be indefinite and so $F_{\infty}$ is isotropic. If $q>2$ is a prime with $q \nmid d$ then $F_{q}$ is unimodular so isotropic. If $q>2$ and $q \mid d$ then $F_{q}$ is isotropic because $\operatorname{det} F= \pm 2 d \neq 1$ in $\mathbb{Q}_{\mathfrak{q}}^{\times} /\left(\mathbb{Q}_{q}^{\times}\right)^{2}$. Finally, if $q=2$ and $p \equiv 3(\bmod 8)$ then $F_{2}$ is isotropic because both $f_{2}(x, y)$ and $u^{2}+2 v^{2}$ represent 3 , while if $p \equiv 7(\bmod 8)$ then $F_{2}$ is isotropic because both $f_{2}(x, y)$ and $u^{2}-2 v^{2}$ represent -1 . 
Let $\left(x_{1}, y_{1}, u_{1}, v_{1}\right) \in \mathbb{Q}^{4} \backslash\{(0,0,0,0)\}$ with $F\left(x_{1}, y_{1}, u_{1}, v_{1}\right)=0$. Since both $f(x, y)$ and $u^{2} \pm 2 v^{2}$ are anisotropic we have $\left(x_{1}, y_{1}\right),\left(u_{1}, v_{1}\right) \neq(0,0)$ so $f\left(x_{1}, y_{1}\right)=u_{1}^{2} \pm 2 v_{1}^{2}=: a^{\prime} \neq 0$. By multiplying $x_{1}, y_{1}, u_{1}, v_{1}$ with a proper rational number we may assume that $x_{1}, y_{1} \in \mathbb{Z}$ and $\left(x_{1}, y_{1}\right)=1$. Hence $f$ represents $a^{\prime}$ primitively, which implies that $f(x, y)=g\left(x^{\prime}, y^{\prime}\right)$, where the mapping $(x, y) \mapsto\left(x^{\prime}, y^{\prime}\right)$ belongs to $\mathrm{SL}(2, \mathbb{Z})$ and $g$ has the form $g\left(x^{\prime}, y^{\prime}\right)=a^{\prime} x^{\prime 2}+b^{\prime} x y+b y^{\prime 2}$ and has the same discriminant as $f$.

Let now $p$ be a prime, $p \nmid a^{\prime}$, with $p \equiv 3(\bmod 4)$ and $\left(\frac{d}{p}\right)=-1$, that is representable by $f$. We write $p=f(x, y)=u^{2} \pm 2 v^{2}$. Then

$$
a^{\prime} p=a^{\prime} g\left(x^{\prime}, y^{\prime}\right)=\left(a^{\prime} x^{\prime}+\frac{b^{\prime}}{2} y^{\prime}\right)^{2}+\frac{4 a^{\prime} c^{\prime}-b^{\prime 2}}{4} y^{\prime 2} .
$$

But $b^{\prime 2}-4 a^{\prime} c^{\prime}=-d$ or $-4 d$, according as $-d \equiv 1(\bmod 4)$ or $-d \equiv 2,3$ $(\bmod 4)$. Hence $p=X^{2}+d Y^{2}$, where $X=a^{\prime} x^{\prime}+\frac{b^{\prime}}{2} y^{\prime}$ and $Y=\frac{1}{2} y^{\prime}$ or $y^{\prime}$, respectively. Note that $X, Y$ are linear combinations of $x^{\prime}, y^{\prime}$, and hence of $x, y$. We also have $a^{\prime} p=\left(u_{1}^{2} \pm 2 v_{1}^{2}\right)\left(u^{2} \pm 2 v^{2}\right)=U^{2} \pm 2 V^{2}$, where $U=u_{1} u \mp v_{1} v$ and $V=u_{1} v+v_{1} u$.

Note that the relations $a^{\prime} p=X^{2}+d Y^{2}=U^{2} \pm 2 V^{2}$ resemble $2 p=$ $x^{2}+5 y^{2}=u^{2} \pm 2 v^{2}$ from $\S 1$. Therefore the reasoning follows the same pattern but with $\mathbb{Q}(\sqrt{5}), 2 p, x \pm y \sqrt{5} i, u \pm v \sqrt{2}$ and $u \pm v \sqrt{2} i$ replaced by $\mathbb{Q}(\sqrt{d}), a^{\prime} p, X \pm Y \sqrt{d} i, U \pm V \sqrt{2}$ and $U \pm V \sqrt{2} i$. Hence we define the fields $F=\mathbb{Q}(\sqrt{d}), E=F(\zeta)=\mathbb{Q}(\sqrt{d}, \sqrt{2}, i)$, where $\zeta:=\zeta_{8}$ and $L=E\left(\sqrt[8]{A_{1}}\right)$, where

$$
A_{1}= \begin{cases}a^{\prime} p(X+Y \sqrt{d} i)^{2}(U+V \sqrt{2} i)^{4} & \text { if } p \equiv 3(\bmod 8), \\ a^{\prime} p(X+Y \sqrt{d} i)^{6}(U+V \sqrt{2})^{4} & \text { if } p \equiv 7(\bmod 8) .\end{cases}
$$

The analogue of Lemma 1.3 holds and, with the notation of $\S 1$, we define again $\chi: \operatorname{Gal}(L / F) \rightarrow \mu_{8}$ by $\sigma^{k} \tau_{l} \mapsto \zeta^{k}$.

Since $\left(\frac{d}{p}\right)=-1, p$ is inert in $F$ and we denote by $\mathfrak{p}$ the only prime of $F$ over $p$. As in $\S 1, \mathfrak{p}$ splits completely in $E$ and we denote by $\mathfrak{P}$ the prime of $E$ over $\mathfrak{p}$ for which $\operatorname{ord}_{\mathfrak{P}}(X-Y \sqrt{d} i)=1$ and $\operatorname{ord}_{\mathfrak{P}}(U-V \sqrt{2} i)=1$ or $\operatorname{ord}_{\mathfrak{P}}(U-V \sqrt{2})=1$, according as $p \equiv 3$ or $7(\bmod 8)$.

By the same proof as for Lemma 1.4(i) we get

$$
\chi\left(\left(\frac{\varepsilon, L / F}{\mathfrak{p}}\right)\right) \equiv \varepsilon^{-\left(p^{2}-1\right) / 8} \quad \text { so } \quad \varepsilon^{\left(p^{2}-1\right) / 8} \equiv \prod_{\mathfrak{q} \neq \mathfrak{p}} \chi\left(\left(\frac{\varepsilon, L / F}{\mathfrak{q}}\right)\right)
$$

modulo $\mathfrak{P}$. So, in principle, the value of $\varepsilon^{\left(p^{2}-1\right) / 8} \bmod \mathfrak{P}$ can be determined and hence we can get $\varepsilon^{\left(p^{2}-1\right) / 8} \bmod p$ by a reasoning similar to that from Lemmas 1.12 and 2.9. The difficulty is that the factors $\chi\left(\left(\frac{\varepsilon, L / F}{\mathfrak{q}}\right)\right)$ with $\mathfrak{q} \neq \mathfrak{p}$ may be $\neq 1$ not only for $\mathfrak{q}=\infty_{ \pm}$or for $\mathfrak{q} \mid 2$ but also for primes $\mathfrak{q} \mid \varepsilon$. It is not clear at this time if in all cases the final answer can be given in terms of $x$ and $y$ alone, as in $\S 1$ and $\S 2$, or if it has to involve also $u$ and $v$. 
The case $d=2$ is different and somewhat easier because if $F=\mathbb{Q}(\sqrt{2})$ and $E=F(\sqrt{2}, \zeta)$ then $\operatorname{Gal}(E / F) \cong \mathbb{Z}_{2}$, unlike the case $d>2$, when $\operatorname{Gal}(E / F) \cong \mathbb{Z}_{8}^{\times} \cong \mathbb{Z}_{2} \times \mathbb{Z}_{2}$. A particular case was conjectured by Z. H. Sun in 1988 (see also [S1, Conjecture 5.1]) and later solved in [S2] and involves the value of $(1+\sqrt{2})^{(p+1) / 4} \bmod p$ for primes $p \equiv 3(\bmod 8)$ and is given in terms of $x, y$, where $p=x^{2}+2 y^{2}$.

The condition that $p \equiv 3(\bmod 4)$ and $\left(\frac{2}{p}\right)=-1$ is equivalent to $p \equiv 3$ $(\bmod 8)$ so it implies that $p=x^{2}+2 y^{2}$ for some $x, y \in \mathbb{Z}$. We define $F=\mathbb{Q}(\sqrt{2}), E=\mathbb{Q}(\zeta)=\mathbb{Q}(\sqrt{2}, i)$ and $L=E(\sqrt[8]{A})$ where $A=p(x+y \sqrt{2} i)^{2}$. The group $\operatorname{Gal}(E / F)$ is generated by the automorphism $\sqrt{2} \mapsto \sqrt{2}, i \mapsto-i$, which coincides with the automorphism $\zeta \mapsto \zeta^{3}$ of $\operatorname{Gal}(E / \mathbb{Q})$. We also have $\operatorname{Gal}(L / E)=\langle\sigma\rangle \cong \mathbb{Z}_{8}$, where $\sigma$ is given by $\sqrt[8]{A} \mapsto \zeta \sqrt[8]{A}$.

\section{Lemma 3.1. The extension $L / F$ is Galois and $\operatorname{Gal}(L / F) \cong \mathbb{Z}_{2} \times \mathbb{Z}_{8}$.}

Proof. We prove that $L / F$ is normal. Let $\alpha=\sqrt[8]{A}$ and let $\beta$ be some conjugate of $\alpha$ over $F$ in some algebraic closure of $F$. Then $\beta^{8}$ is a conjugate of $\alpha^{8}=A$ over $F$ so $\beta^{8} \in\left\{A, A^{\prime}\right\}$, where $A^{\prime}=p(x-y \sqrt{2} i)^{2}$. Since $p=(x+y \sqrt{2} i)(x-y \sqrt{2} i)$ we have

$$
A^{\prime}=p^{3}(x+y \sqrt{2} i)^{-2}=A^{3}(x+y \sqrt{2} i)^{-8}=\alpha^{\prime 8},
$$

where $\alpha^{\prime}=\alpha^{3}(x+y \sqrt{2} i)^{-1} \in L$. If $\beta^{8}=A$ then $\beta=\zeta^{k} \alpha \in L$, while if $\beta^{8}=A^{\prime}$ then $\beta=\zeta^{k} \alpha^{\prime} \in L$ for some $k$. So $L / F$ is normal.

Let now $\phi \in \operatorname{Gal}(L / F)$. Then $\phi_{\mid E} \in \operatorname{Gal}(E / F)$ so is given by $\zeta \mapsto \zeta$ or $\zeta \mapsto \zeta^{3}$. If $\phi(\zeta)=\zeta$ then $\phi(\alpha)^{8}=\phi(A)=A$ so $\phi(\alpha)=\zeta^{k} \alpha$, i.e. $\phi=\sigma^{k}$ for some $k$. If $\phi(\zeta)=\zeta^{3}$ then $\phi(\alpha)^{8}=\phi(A)=A^{\prime}$ and hence $\phi(\alpha)=\zeta^{k} \alpha^{\prime}$ for some $k$. So the 16 automorphisms of $L$ over $F$ are given by $\zeta \mapsto \zeta$ and $\alpha \mapsto \zeta^{k} \alpha$ or $\zeta \mapsto \zeta^{3}$ and $\alpha \mapsto \zeta^{k} \alpha^{\prime}$ with $k \in \mathbb{Z}_{8}$. We denote by $\tau$ the automorphism $\zeta \mapsto \zeta^{3}, \alpha \mapsto \alpha^{\prime}$.

We claim that $\langle\tau\rangle \cong \mathbb{Z}_{2}$ and $\operatorname{Gal}(L / F)$ is the internal direct product of $\langle\tau\rangle$ and $\operatorname{Gal}(L / E)=\langle\sigma\rangle$ and so it is isomorphic to $\mathbb{Z}_{2} \times \mathbb{Z}_{8}$. We have to show that $\tau^{2}=1, \tau \sigma=\sigma \tau$ and $\langle\tau\rangle \cap\langle\sigma\rangle=\{1\}$. For the first condition we have $\tau(\zeta)=\zeta^{3}$ so $\tau^{2}(\zeta)=\zeta^{9}=\zeta$ and

$$
\begin{aligned}
\tau^{2}(\alpha) & =\tau\left(\alpha^{\prime}\right)=\tau\left(\alpha^{3}(x+y \sqrt{2})^{-1}\right)=\alpha^{\prime 3}(x-y \sqrt{2} i)^{-1} \\
& =\alpha^{9}(x+y \sqrt{2})^{-3}(x-y \sqrt{2} i)^{-1}=\alpha p(x+y \sqrt{2} i)^{-1}(x-y \sqrt{2} i)^{-1}=\alpha .
\end{aligned}
$$

(Recall that $\alpha^{8}=A=p(x+y \sqrt{2} i)^{2}$.) So $\tau^{2}=1$. For the second condition $\tau \sigma(\zeta)=\tau(\zeta)=\zeta^{3}$ and $\sigma \tau(\zeta)=\sigma\left(\zeta^{3}\right)=\zeta^{3}$. Also

$$
\begin{aligned}
& \tau \sigma(\alpha)=\tau(\zeta \alpha)=\zeta^{3} \alpha^{\prime}=\zeta^{3} \alpha^{3}(x+y \sqrt{2} i)^{-1}, \\
& \sigma \tau(\alpha)=\sigma\left(\alpha^{\prime}\right)=\sigma\left(\alpha^{3}(x+y \sqrt{2} i)^{-1}\right)=\zeta^{3} \alpha^{3}(x+y \sqrt{2} i)^{-1} .
\end{aligned}
$$


So $\tau \sigma=\sigma \tau$. Finally, the third condition follows from the fact that $\tau(\zeta) \neq$ $\zeta$ so $\tau \notin \operatorname{Gal}(L / E)=\langle\sigma\rangle$.

We define $\chi: \operatorname{Gal}(L / F) \rightarrow \mu_{8}$ by $\sigma^{k} \tau_{l} \mapsto \zeta^{k}$. As in $\S 1, \chi(\phi)=\phi(\alpha) / \alpha$ if $\phi \in\langle\sigma\rangle=\operatorname{Gal}(L / E)$.

Since $\left(\frac{2}{p}\right)=-1$ we see that $p$ is inert in $F$. Let $\mathfrak{p}$ be the prime of $F$ over $p$. Since $\left(\frac{-2}{p}\right)=1$ we infer that -2 is a square in $\mathbb{Q}_{p}$ and so in $F_{\mathfrak{p}}$. Thus $\mathfrak{p}$ splits in $E$. Since $(x+y \sqrt{2} i)(x-y \sqrt{2} i)=p$ each of $x \pm y \sqrt{2} i$ belongs to one of the two primes of $E$ over $\mathfrak{p}$. We denote by $\mathfrak{P}$ the prime of $E$ over $p$ such that $x-y \sqrt{2} i \in \mathfrak{P}$.

By the same proof as for Lemma 1.4(i) (with $\alpha$ instead of $\alpha_{1}$ ) we get $\chi\left(\left(\frac{\varepsilon, L / F}{\mathfrak{p}}\right)\right) \equiv \varepsilon^{-\left(p^{2}-1\right) / 8}(\bmod \mathfrak{P})$ and then we follow the same reasoning as in the case $d>2$.

We have $\chi\left(\left(\frac{\varepsilon, L / F}{\mathfrak{p}}\right)\right) \in \mu_{8}$ so it has the form $\alpha, \beta i$ or $(\alpha+\beta i) / \sqrt{2}$ with $\alpha, \beta= \pm 1$. The value of $\varepsilon^{-\left(p^{2}-1\right) / 8} \bmod p$ can be found from $\varepsilon^{-\left(p^{2}-1\right) / 8} \bmod \mathfrak{P}$ by using the following lemma.

Lemma 3.2. Let $s, t$ be $p$-adic integers and $\alpha, \beta \in\{ \pm 1\}$.

(i) If $s+t \sqrt{2} \equiv \alpha(\bmod \widetilde{\mathfrak{P}})$ then $s \equiv \alpha(\bmod p)$ and $t \equiv 0(\bmod p)$.

(ii) If $s+t \sqrt{2} \equiv \beta i(\bmod \widetilde{\mathfrak{P}})$ then $s \equiv 0(\bmod p)$ and $t \equiv-\beta \frac{y}{x}(\bmod p)$.

(iii) If $s+t \sqrt{2} \equiv \frac{\alpha+\beta i}{\sqrt{2}}(\bmod \widetilde{\mathfrak{P}})$ then $s \equiv-\beta \frac{y}{x}(\bmod p)$ and $t \equiv \frac{1}{2} \alpha$ $(\bmod p)$.

Proof. We have $x-y \sqrt{2} i \in \widetilde{\mathfrak{P}}$ so $x \equiv y \sqrt{2} i(\bmod \widetilde{\mathfrak{P}})$. Since $p \nmid x y$ we get $i \equiv-\frac{y}{x} \sqrt{2}(\bmod \widetilde{\mathfrak{P}})$ and $\frac{i}{\sqrt{2}} \equiv-\frac{y}{x}(\bmod \widetilde{\mathfrak{P}})$. It follows that $\beta i \equiv-\beta \frac{y}{x} \sqrt{2}$ $(\bmod \widetilde{\mathfrak{P}})$ and

$$
\frac{\alpha+\beta i}{\sqrt{2}}=\frac{1}{2} \alpha \sqrt{2}+\beta \frac{i}{\sqrt{2}} \equiv \frac{1}{2} \alpha \sqrt{2}-\beta \frac{y}{x}(\bmod \widetilde{\mathfrak{P}}) .
$$

Therefore the congruences from the hypotheses of (i)-(iii) can be written as $s+t \sqrt{2} \equiv \alpha,-\beta \frac{y}{x} \sqrt{2}$ or $-\beta \frac{y}{x}+\frac{1}{2} \alpha \sqrt{2}(\bmod \widetilde{\mathfrak{P}})$, respectively. Since both sides of these congruences belong to $F_{\mathfrak{p}}$ they will also hold modulo $\widetilde{\mathfrak{p}}$.

\section{References}

[G] R. K. Guy, Unsolved Problems in Number Theory, 2nd ed., Springer, New York, 1994.

[L] F. Lemmermeyer, Reciprocity Laws: From Euler to Eisenstein, Springer, Berlin, 2000.

[S1] Z. H. Sun, Values of Lucas sequences modulo primes, Rocky Mountain J. Math. 33 (2003), 1123-1145. 
[S2] Z. H. Sun, Quartic, octic residues and Lucas sequences, preprint.

[SS] Z. H. Sun and Z. W. Sun, Fibonacci numbers and Fermat's last theorem, Acta Arith. 60 (1992), 371-388.

Institute of Mathematics "Simion Stoilow"

of the Romanian Academy

21 Calea Grivitei St.

010702 Bucureşti, Sector 1, Romania

E-mail: Constantin.Beli@imar.ro, raspopitu1@yahoo.com

Received on 9.4.2007

and in revised form on 1.10.2008 